\title{
Vetoing cosmogenic muons in a large liquid scintillator
}

\author{
Marco Grassi, ${ }^{a, b}$ Jarah Evslin, ${ }^{c}$ Emilio Ciuffoli ${ }^{c}$ and Xinmin Zhang ${ }^{d, e}$ \\ ${ }^{a}$ Institute of High Energy Physics (IHEP), CAS, \\ YuQuan Lu 19B, Beijing 100049, China \\ ${ }^{b}$ INFN Sezione di Milano, \\ via Celoria 16, 20133 Milan, Italy \\ ${ }^{c}$ Institute of Modern Physics, CAS, \\ NanChangLu 509, Lanzhou 730000, China \\ ${ }^{d}$ Theoretical Physics Division, IHEP, CAS, \\ YuQuan Lu 19B, Beijing 100049, China \\ e Theoretical Physics Center for Science Facilities, IHEP, CAS, \\ YuQuan Lu 19B, Beijing 100049, China \\ E-mail: mgrassi@ihep.ac.cn, jarah@impcas.ac.cn, emilio@impcas.ac.cn, \\ xmzhang@ihep.ac.cn
}

ABSTRACT: At upcoming medium baseline reactor neutrino experiments the spallation ${ }^{9} \mathrm{Li}$ background will be somewhat larger than the inverse $\beta$ decay reactor neutrino signal. We use new FLUKA simulations of spallation backgrounds to optimize a class of veto strategies and find that surprisingly the optimal veto for the mass hierarchy determination has a rejection efficiency below $90 \%$. The unrejected background has only a modest effect on the physics goals. For example $\Delta \chi^{2}$ for the hierarchy determination falls by 1.4 to 3 points depending on the muon tracking ability. The optimal veto strategy is essentially insensitive to the tracking ability, consisting of 2 meter radius, 1.1 second cylindrical vetoes of well tracked muons with showering energies above 3 to $4 \mathrm{GeV}$ and 0.7 second full detector vetoes for poorly tracked muons above 15 to $18 \mathrm{GeV}$. On the other hand, as the uncertainty in $\theta_{12}$ will be dominated by the uncertainty in the reactor neutrino spectrum and not statistical fluctuations, the optimal rejection efficiency for the measurement of $\theta_{12}$ is $93 \%$ in the case of perfect tracking.

KeYwords: Neutrino Detectors and Telescopes, Oscillation

ARXIV EPRINT: 1505.05609 


\section{Contents}

1 Introduction 1

2 The ${ }^{9} \mathrm{Li}$ background $\quad 2$

2.1 The distribution of ${ }^{9} \mathrm{Li}$

3 Live time vs rejection efficiency $\quad 5$

3.1 The veto strategy 5

3.2 Full detector veto 6

$\begin{array}{lll}3.3 & \text { Cylindrical veto } & 7\end{array}$

4 Optimal veto for each science goal 10

$\begin{array}{lll}4.1 & \text { Neutrino mass hierarchy } & 11\end{array}$

$\begin{array}{ll}4.2 \text { Uncertainty in the }{ }^{9} \mathrm{Li} \text { spectrum } & 13\end{array}$

$\begin{array}{lll}4.3 & \text { Mixing angle } \theta_{12} & 14\end{array}$

5 Imperfect tracking $\quad \mathbf{1 6}$

$\begin{array}{ll}5.1 \text { The neutrino mass hierarchy } & 18\end{array}$

$\begin{array}{ll}5.2 \text { Measuring } \theta_{12} & 20\end{array}$

6 Conclusions $\quad 22$

\section{Introduction}

The next generation of medium baseline reactor neutrino experiments [1, 2] will deliver by far the most precise measurement yet of $\theta_{12}$ [3] and also will be sensitive to the neutrino mass hierarchy $[4,5]$. Cosmogenic muons interacting with ${ }^{12} \mathrm{C}$ in their organic liquid scintillator detectors will create spallation products ${ }^{9} \mathrm{Li}$ and ${ }^{8} \mathrm{He}$ whose decays yield the same double coincidence used to detect reactor neutrinos [6]. The background will be larger than the signal [7]. The effectiveness of a veto strategy depends critically on the ability to track these muons, so that only events near the track need be vetoed. In this note we present the optimal strategy as a function of the tracking efficiency. Our main result is that the optimal strategy leads to the acceptance of a much larger fraction of the background than had been found in previous studies but that this large background has a relatively minor effect on the main science goals of these experiments.

On a smaller scale, this issue has already been confronted by KamLAND [8]. As the detector is deeper and smaller, the muon rate is only $0.2 \mathrm{~Hz}$, appreciably slower than the $257 \mathrm{~ms}$ decay time of ${ }^{9} \mathrm{Li}$. Of these muons, the most dangerous are the showering muons. These are defined to be muons which deposit at least $3 \mathrm{GeV}$ in the detector in addition to the energy deposited by ionization. While they occur at a rate of only $0.03 \mathrm{~Hz}$, they are 
responsible for the majority of the ${ }^{9} \mathrm{Li}$ background [9]. As a result, KamLAND chose a full detector veto of 2 seconds after all showering muons and also after muons whose track could not be reconstructed. For nonshowering muons whose track could be reconstructed, a 3 meter radius cylinder about the track was vetoed for 2 seconds. The combination of these vetoes left a small number of background events, estimated for example as 14 [10] of the original $10^{3}$ [8]. Overall, this strategy led to a rejection efficiency of about $99 \%$ while removing only about $15 \%$ of the signal.

Can this same strategy be repeated with upcoming experiments like JUNO and RENO 50 ? JUNO will have a muon rate of about $5 \mathrm{~Hz}$ and a showering muon rate of about 1 $\mathrm{Hz}$, and so a 2 second veto after each showering muon would eliminate essentially all of the events [7]. However we will show in this paper that a much less efficient veto can give an acceptable dead time and result in a relatively modest effect on the main science goals of the experiment, the determination of the neutrino mass hierarchy and the most accurate ever measurement of $\theta_{12}$. The reason for this is that the shape and normalization of the ${ }^{9} \mathrm{Li}$ decay spectrum can be determined fairly well, and the ${ }^{8} \mathrm{He}$ decays yield a small background. Therefore the background can be subtracted, leaving only a small contribution to the statistical fluctuations. In this note we will determine the optimal veto strategy and its impact on the sensitivity to the hierarchy and the measurement of $\theta_{12}$.

This procedure is somewhat complicated by muon bundles. At both KamLAND and JUNO, nearly half of the muons that hit the detector are contained in bundles of multiple muons created in the same cosmic ray collision. However, as the KamLAND detector is much smaller than the characteristic muon separation, in less than $5 \%$ of events do multiple muons from the same bundle strike the detector. On the other hand, in the case of JUNO, about $10 \%$ of muon events will consist of multiple muons striking the detector. The tracking of these bundle events is extremely challenging, and so the ability to veto only the muon track, and not the full detector, will depend on whether JUNO is capable of tracking multiple muons. In this study we will examine the dependence of the sensitivity to the hierarchy and the precision with which $\theta_{12}$ can be measured upon the tracking ability of the detector.

\section{The ${ }^{9} \mathrm{Li}$ background}

$51 \%$ of ${ }^{9} \mathrm{Li}$ and $16 \%$ of ${ }^{8} \mathrm{He}$ decays yield a neutron together with an electron, mimicking the double coincidence signal of inverse $\beta$ decay (IBD). At JUNO these double coincidence background rates will be $8.5 \times 10^{-4} \mathrm{~Hz}$ and $3.0 \times 10^{-5} \mathrm{~Hz}$ respectively. The two decay spectra are so similar that so far KamLAND has not been able to conclusively observe ${ }^{8} \mathrm{He}$ beyond the $1 \sigma$ level. Therefore, to a reasonable approximation the ${ }^{8} \mathrm{He}$ background may be ignored.

\subsection{The distribution of ${ }^{9} \mathrm{Li}$}

At KamLAND a full detector or cylindrical veto was applied to all cosmogenic muons. However those muons whose showering energy, defined to be the energy that they deposit in the scintillator minus $1.43 \mathrm{MeV}$ per $\mathrm{cm}$ of their track, ${ }^{1}$ is sufficiently small are exceedingly

\footnotetext{
${ }^{1} 1.43 \mathrm{MeV} / \mathrm{cm}$ is the ionization energy deposited in electrons with less than $100 \mathrm{keV}$. Those with more energy are considered to be $\delta$ rays and little would be gained by subtracting them as well from the showering energy [7].
} 


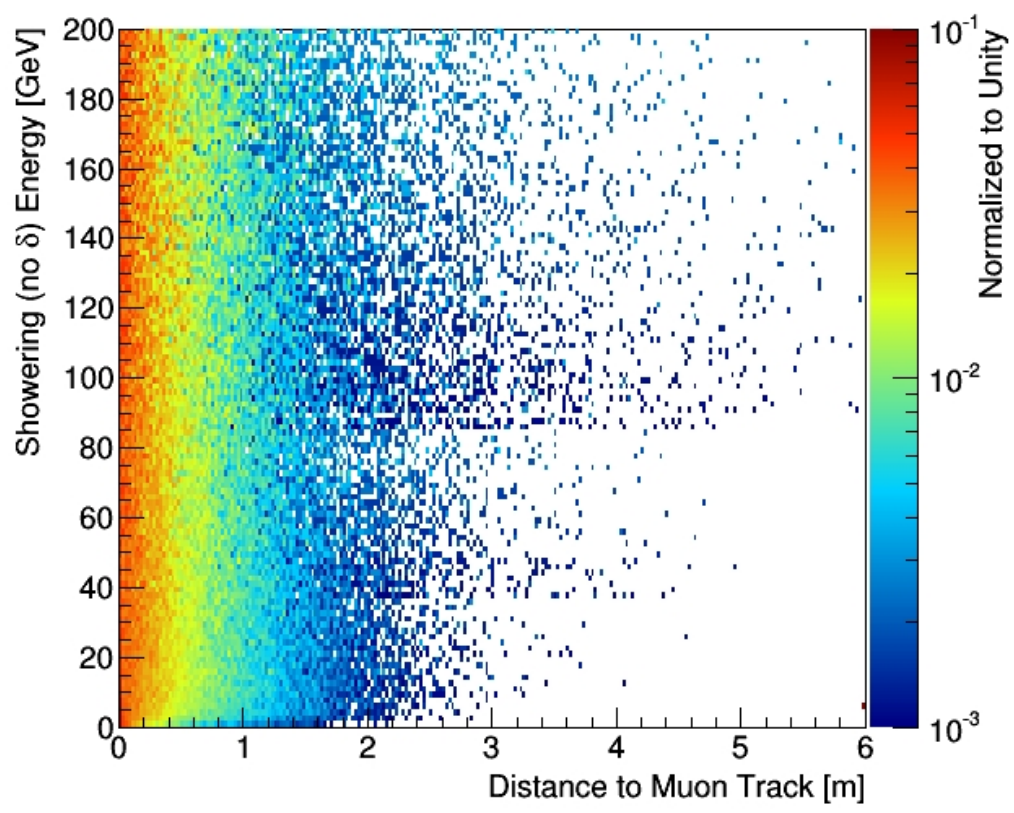

Figure 1. The radial distribution (horizontal) of ${ }^{9} \mathrm{Li}$ about a muon track, normalized to unity for each muon showering energy (vertical).

unlikely to generate any ${ }^{9} \mathrm{Li}$. In fact, we will see below that in the case of JUNO, there are so many muons that the optimal strategy is not to apply any veto to muons whose showering energy is less than a certain value, which is at least about $3 \mathrm{GeV}$ in each case. The average muon track length is about 24 meters. To determine the volume-weighted dead time, we will need the average track length of only those muons whose showering energies exceed a certain minimum $E_{t}$. We have found that this average track length is 28 meters whenever $E_{t}>1.5 \mathrm{GeV}$, as it for the veto strategies that we will find to be optimal. Therefore, while we will use the average track length $d\left(E_{t}\right)$ as determined by our FLUKA simulations, in practice for the optimal veto strategies this will always be equal to 28 meters.

The distribution of ${ }^{9} \mathrm{Li}$ events about this track then depends on the muon's showering energy and also the distance from the muon track. As can be seen in figure 1, this distribution to a very good approximation factorizes, in the sense that the radial distribution of ${ }^{9} \mathrm{Li}$ is essentially independent of the showering energy. This greatly simplifies our analysis, as it means that the ${ }^{9} \mathrm{Li}$ yield is entirely characterized by just two functions, the radial, shown in figure 2 and showering energy distribution which was already provided in ref. [7].

In the next sections we will attempt to determine the optimal veto strategy. To do this, we will need the fraction of muons with a showering energy above a threshold $E_{t}$ and the fraction of ${ }^{9} \mathrm{Li}$ produced by those muons. These are displayed respectively in figures 3 and 4. 


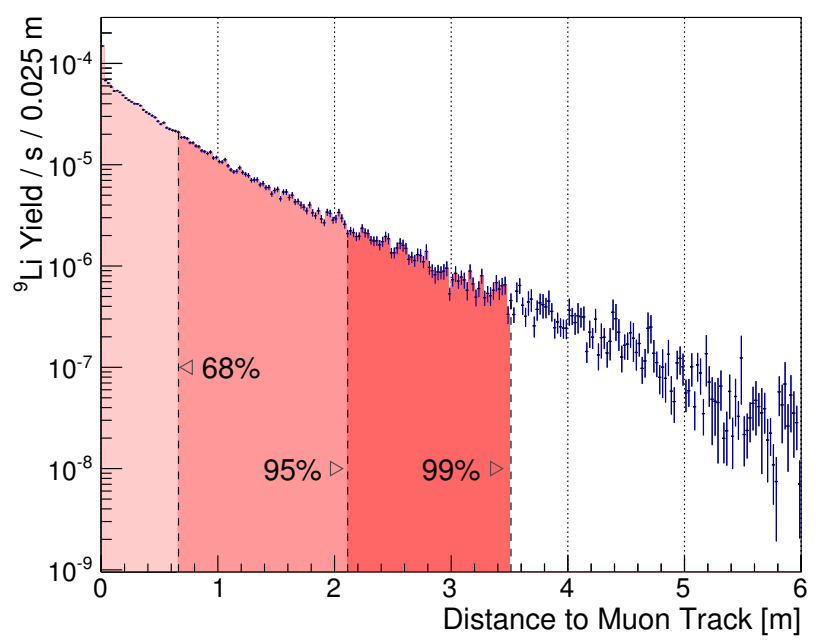

Figure 2. The radial distribution of ${ }^{9} \mathrm{Li}$ about a muon track.

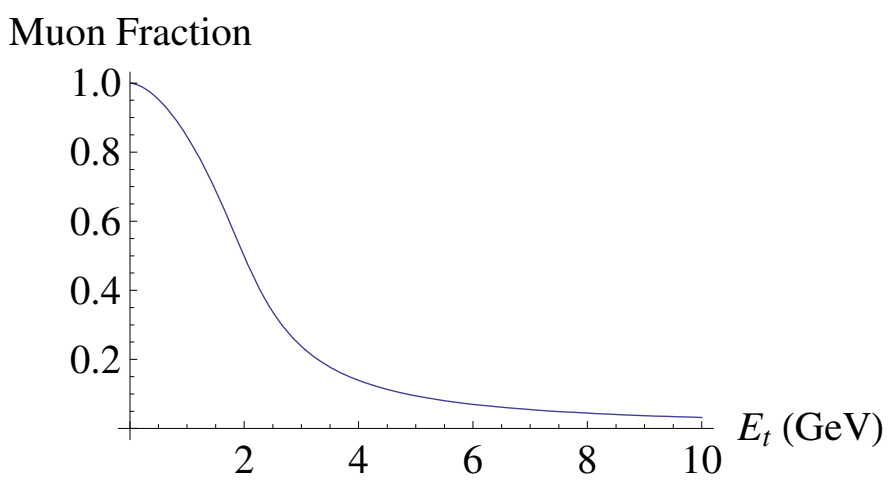

Figure 3. The cumulative fraction of muons with a showering energy above the threshold given by $E_{t}$ (the $x$-axis).
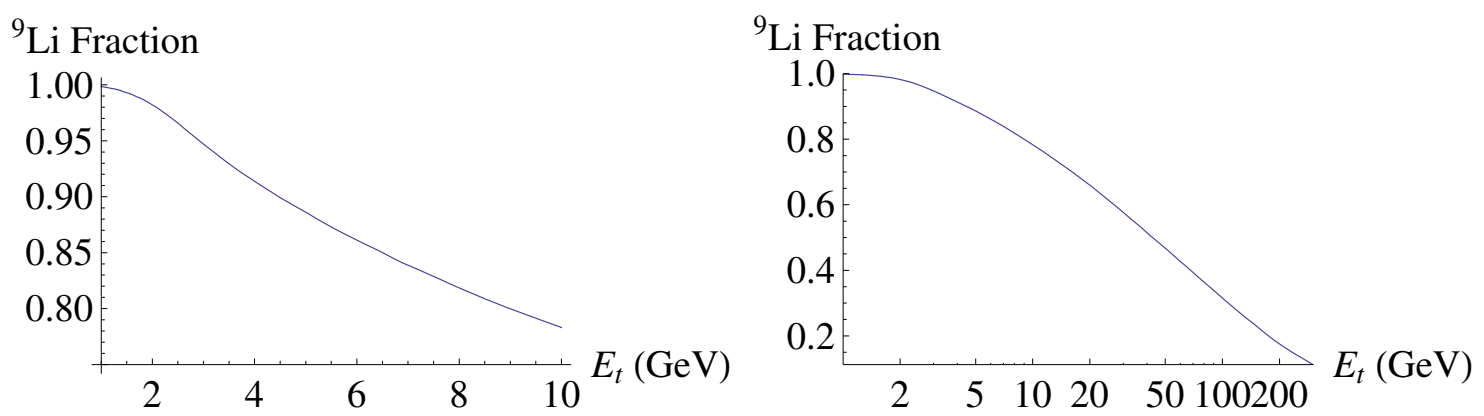

Figure 4. The cumulative fraction of ${ }^{9} \mathrm{Li}$ generated by muons with a showering energy above the threshold $E_{t}$ (the $x$-axis). 


\section{$3 \quad$ Live time vs rejection efficiency}

\subsection{The veto strategy}

In practice the optimal veto strategy may be quite complicated, and any strategy should be refined once data taking begins. Our goal in this paper is to understand the effect of the spallation isotope backgrounds on the science goals of medium baseline reactor experiments and the implications of these backgrounds for the muon tracking requirements. As a result, it is necessary to produce a reasonably effective but simple veto strategy, which can be used in the analysis.

The strategies that we will consider consist of two cuts

1) When the energy $E>E_{f}$ and it is known that the muon (bundle) was not tracked successfully, veto the full detector for a time $t_{f}$.

2) When the $E>E_{c}$ and it is believed that the muon was tracked successfully, veto a cylinder of radius $r_{c}$ about each muon track for a time $t_{c}$.

We will optimize $E_{f}, E_{c}, r_{c}, t_{f}$ and $t_{c}$ separately for the neutrino mass hierarchy determination and for the measurement of $\theta_{12}$. We will first assume that each muon is tracked perfectly. This assumption will be relaxed in section 5 .

One might think that the simultaneous optimization of these 5 variables would require a slow, global search through a 5-dimensional space, rerunning our simulations at each step. In fact the simulations only depend on two parameters, the background rejection efficiency and the live time. Therefore we will use the following strategy. First, in this section, using the results of section 2.1 we will find the strategy which maximizes the live time for each possible background rejection efficiency. This maximum is independent of the science goal considered, and so this section will apply to both the hierarchy search and to $\theta_{12}$, and can in the future be applied to other potential science goals such as the study of geoneutrinos should the reactors all be turned off in the future.

Next, in sections 4 and 5 simulations of the detector JUNO with respectively perfect and imperfect tracking are considered with each possible background rejection efficiency and live time. As the optimal live time for each detector efficiency will already be determined, for each science goal we will only need to optimize a single variable, the background rejection efficiency. This will yield the optimal cuts as well as the effect of the background upon the hierarchy determination and the measurement of $\theta_{12}$.

Let $m(E)$ and $l(E)$ respectively be the fraction of single muons and the fraction of ${ }^{9} \mathrm{Li}$ isotopes created by single muons with showering energy greater than $E$. These two functions, in the case of the detector JUNO are plotted in figures 3 and 4 . Here we have not considered muon bundle events. While only $10 \%$ of muon events at JUNO will be multimuon events, these will account for about a third of all muons and so about a third of spallation isotope production.

In the case of muon bundles, the spallation isotope production rate is just the sum of the rates for the individual muons. The energy distribution for muon bundles is similar to that of single muons and so an extension of our analysis to bundle events would be 


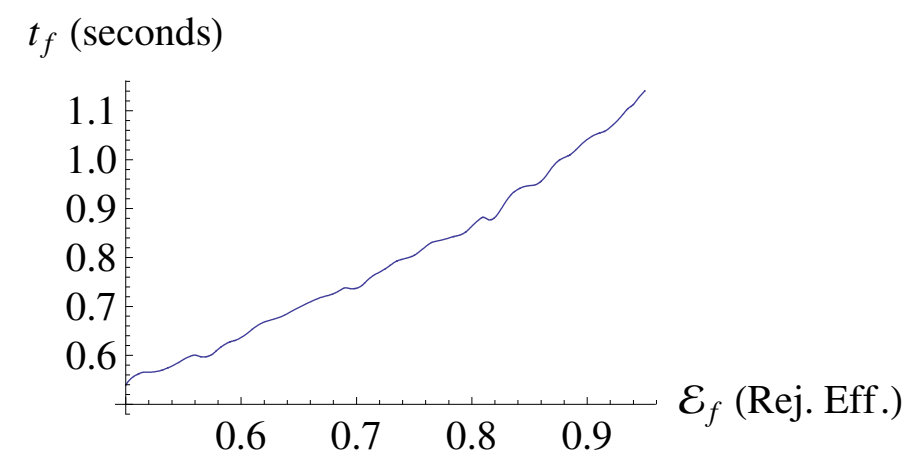

Figure 5. The optimal full detector veto time $t_{f}\left(\mathcal{E}_{f}\right)$ yielding an ${ }^{9} \mathrm{Li}$ rejection efficiency $\mathcal{E}_{f}$.

straightforward. One need only modify the showering energy distribution $m(E)$ and spallation isotope distribution $l(E)$ functions to be the weighted sum of the distributions for all $k$-muon bundles, where the total deposited energy is the total of that of the $k$ muons. This requires no new simulations, as the $k$-bundle distribution is essentially just the sum of $k$ single muon distributions. One only needs to know the probability of multimuon events, which in the case $k=2$ is calculated in ref. [7].

The inclusion of bundles would reduce the rejection efficiency as the scintillator detectors cannot determine how much energy was deposited by each muon, and so one would need to rely upon a total energy threshold. As a result, the rejection efficiencies obtained in this study are optimistic estimates. However, it will be clear below that even a $10 \%$ reduction in the rejection efficiency would not have an enormous impact on the science goals.

\subsection{Full detector veto}

A full detector veto of time $t_{f}$ after the passage of each muon with a showering energy of at least $E_{f}$ will yield a rejection efficiency of

$$
\mathcal{E}_{f}\left(E_{f}, t_{f}\right)=l\left(E_{f}\right)\left(1-e^{-t_{f} / t_{d}}\right)
$$

where $t_{d} \sim 255 \mathrm{~ms}$ is the decay time for ${ }^{9} \mathrm{Li}$. Only $\beta$ decays of ${ }^{9} \mathrm{Li}$ to excited states of ${ }^{9}$ Be eventually yield free neutrons and so a false double coincidence, however the decay of ${ }^{9} \mathrm{Be}$ into two $\alpha$ particles and a neutron is much faster than the $\beta$ decay of ${ }^{9} \mathrm{Li}$ and the $\beta$ decay time of ${ }^{9} \mathrm{Li}$ is independent of whether the ${ }^{9} \mathrm{Be}$ is in an excited state, and so $t_{f}$ for the decays producing neutrons is equal to the overall $t_{f}$ for ${ }^{9} \mathrm{Li}$.

For a given rejection efficiency $\mathcal{E}_{f}$, one can invert (3.1) to obtain $E_{f}$ as a function of the veto time $t_{f}$

$$
E_{f}\left(\mathcal{E}_{f}, t_{f}\right)=l^{-1}\left(\frac{\mathcal{E}_{f}}{1-e^{-t_{f} / t_{d}}}\right)
$$

where $l^{-1}$ is the inverse of the function $l(E)$ defined in eq. (3.1). The resulting fractional live time $t_{l}$ is determined from the product of the veto rate $m\left(E_{f}\right)$ and the veto time $t_{f}$

$$
t_{l}\left(\mathcal{E}_{f}, t_{f}\right)=\exp \left(-t_{f} m\left(E_{f}\left(\mathcal{E}_{f}, t_{f}\right)\right)\right)
$$



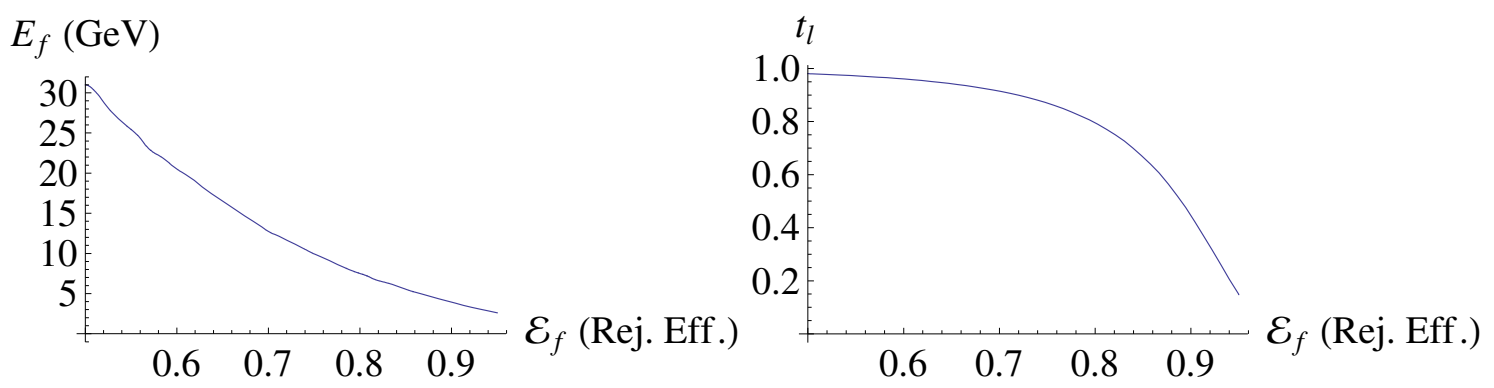

Figure 6. The optimal full detector showering energy threshold $E_{f}\left(\mathcal{E}_{f}\right)$ and fractional live time $t_{l}\left(\mathcal{E}_{f}\right)$ yielding an ${ }^{9} \mathrm{Li}$ rejection efficiency $\mathcal{E}_{f}$.

The veto may then be optimized, for each $\mathcal{E}_{f}$, by simply setting

$$
\frac{\partial t_{l}\left(\mathcal{E}_{f}, t_{f}\right)}{\partial t_{f}}=0
$$

The solution to this equation is the optimal $t_{f}$ for each rejection efficiency $\mathcal{E}_{f}$, which we will call $t_{f}\left(\mathcal{E}_{f}\right)$. The optimal veto time $t_{f}\left(\mathcal{E}_{f}\right)$ for each efficiency $\mathcal{E}_{f}$ is shown in figure 5 . Note that the optimal veto time is about 1 second, much less than the 2 seconds at KamLAND.

Once $t_{f}\left(\mathcal{E}_{f}\right)$ is found numerically, $E_{f}\left(\mathcal{E}_{f}\right)$ is found using eq. (3.2) and the fractional live time $t_{l}\left(\mathcal{E}_{f}\right)$ using (3.3). These are shown in figure 6 . Note that even a modest rejection efficiency of $90 \%$ requires a showering energy threshold of about $4 \mathrm{GeV}$ and leads to a live time of less than one half of the total runtime. In a 20 kton detector, full detector vetoes are quite costly. As a result, we will assume that they are only used when the muon was not tracked successfully, a case which will be considered in section 5 . In section 4 we will assume that all muons are well tracked and so only cylindrical vetoes will be considered.

\subsection{Cylindrical veto}

The efficiency of a cylindrical veto depends not only on the functions $m(E)$ and $l(E)$ characterizing the muon distribution and ${ }^{9} \mathrm{Li}$ yields as functions of energy $E$, but also upon the ${ }^{9} \mathrm{Li}$ radial distribution about the muon track $f(R)$. Here $f(R)$ is the ${ }^{9} \mathrm{Li}$ rate per unit radius at a radial distance $R$ from the track, as illustrated in figure 2. A radius $r_{c}$ cylindrical veto of time $t_{c}$ after the passage of each muon with a showering energy of at least $E_{c}$ will yield a rejection efficiency of

$$
\mathcal{E}_{c}\left(E_{c}, t_{c}, r_{c}\right)=l\left(E_{c}\right)\left(1-e^{-t_{c} / t_{d}}\right) \frac{\int_{0}^{r_{c}} f(R) d R}{\int_{0}^{\infty} f(R) d R} .
$$

For simplicity we will define the integral

$$
F\left(r_{c}\right)=\frac{\int_{0}^{r_{c}} f(R) d R}{\int_{0}^{\infty} f(R) d R}
$$

which is equal to the normalized ${ }^{9} \mathrm{Li}$ event rate at radii below $r_{c}$. 

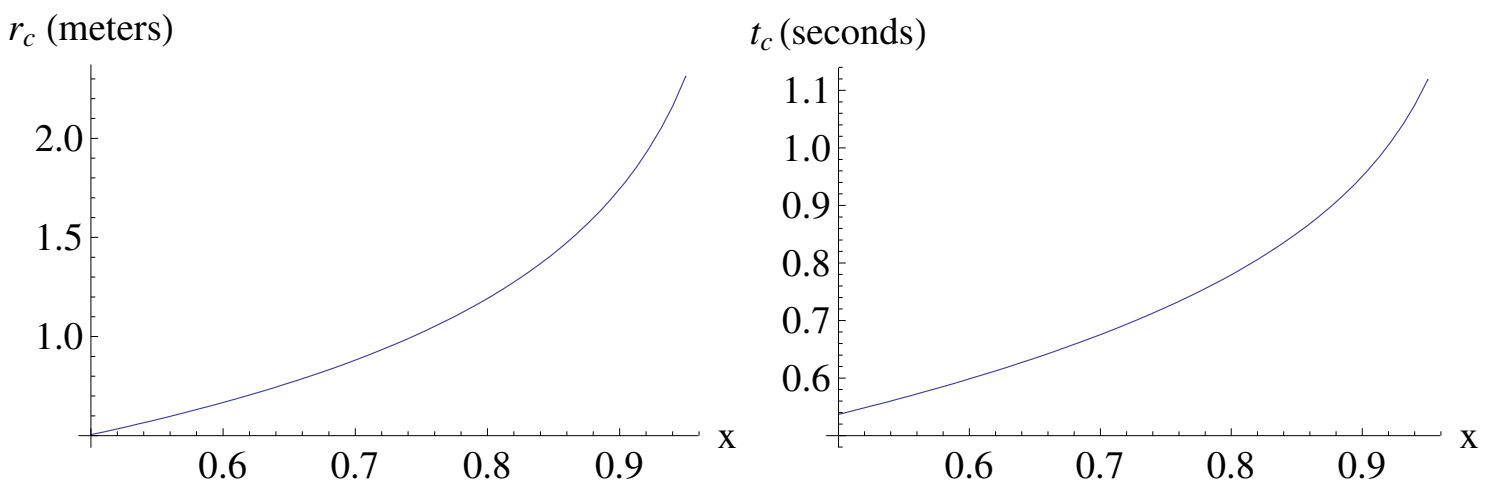

Figure 7. The optimal cylindrical veto radius $r_{c}$ and time $t_{c}$ for a fixed value of $x$, defined in eq. (3.7).

Note that the expression (3.5) for the rejection efficiency $\mathcal{E}_{c}$ factorizes into a part dependent upon $E_{c}$ and a part $x$ dependent upon $t_{c}$ and $r_{c}$

$$
\mathcal{E}_{c}\left(E_{c}, t_{c}, r_{c}\right)=l\left(E_{c}\right) x\left(t_{c}, r_{c}\right), \quad x\left(t_{c}, r_{c}\right)=\left(1-e^{-t_{c} / t_{d}}\right) F\left(r_{c}\right) .
$$

Therefore we may separate the optimization problem into steps. First we may fix $x$. This gives a relation between $t_{c}$ and $r_{c}$

$$
t_{c}\left(x, r_{c}\right)=-t_{d} \ln \left(1-\frac{x}{F\left(r_{c}\right)}\right) .
$$

Now instead of the three independent variables $\left(E_{c}, t_{c}, r_{c}\right)$ there are three independent variables $\left(E_{c}, r_{c}, x\right)$. However the rejection efficiency $\mathcal{E}_{c}$ is entirely determined by $E_{c}$ and $x$ via eq. (3.7) and so $r_{c}$ can be varied, fixing $E_{c}$ and $x$, so as to minimize the total dead time. Here the volume-weighted dead time that is relevant is the veto time multiplied by the veto volume corresponding to a live time of

$$
t_{l}\left(E_{c}, r_{c}, x\right)=\exp \left(-\frac{\pi d\left(E_{c}\right) r_{c}^{2}}{V} t_{c}\left(x, r_{c}\right) m\left(E_{c}\right)\right)
$$

where $V$ is the detector volume and, following the definition in section 2.1, $d\left(E_{c}\right)$ is the average tracklength of a muon with showering energy $E_{c}$. The detector efficiency will be proportional to $t_{l}$.

We want to choose $r_{c}$ so as to maximize $t_{l}$. However $r_{c}$ only enters in the combination $r_{c}^{2} t_{c}\left(x, r_{c}\right)$ and so we only need minimize $r_{c}^{2} t_{c}\left(x, r_{c}\right)$ for each value of $\mathrm{x}$

$$
\frac{\partial r_{c}^{2} t_{c}\left(x, r_{c}\right)}{\partial r_{c}}=0
$$

Using eq. (3.8) this minimization is easily performed, yielding the optimal value of $r_{c}(x)$ and therefore $t_{c}(x)$ for each value of $x$. The optimal values are shown in figure 7. Similarly to the full detector vetoes, the optimal veto time is about 1 second, only half as long as the KamLAND vetoes. Similarly the optimal veto radius is always less than the 3 meter KamLAND 


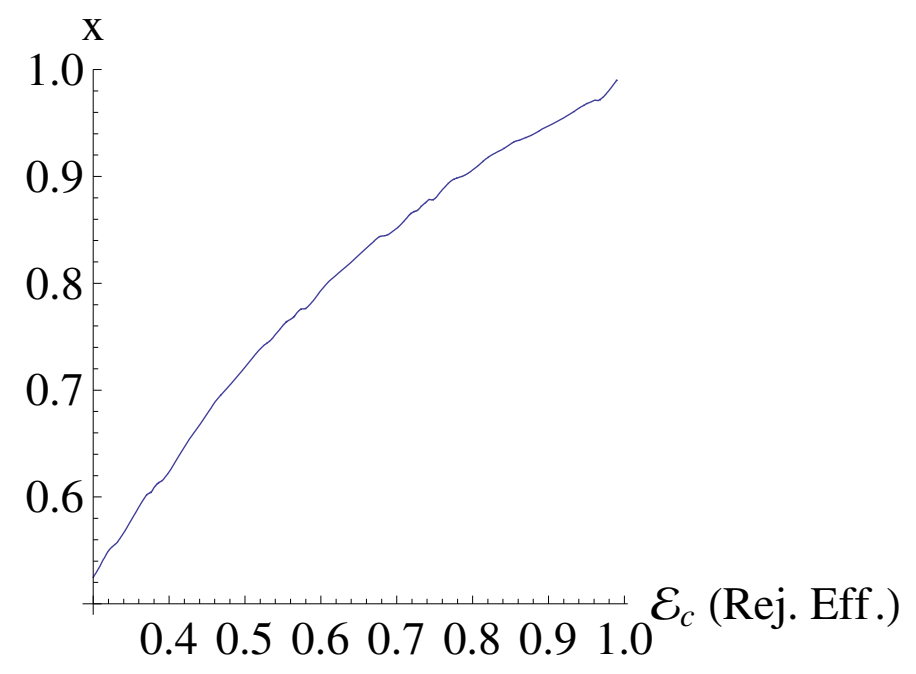

Figure 8. The optimal value of $x$, defined in eq. (3.7), as a function of the efficiency $\mathcal{E}_{c}$ of a cylindrical veto.

veto. Due to KamLAND's greater depth and smaller size, the muon rate is much lower which allows KamLAND to use longer vetoes with a small effect on the measurement of the reactor mixing parameters. While an analysis such as ours applied to KamLAND would nonetheless find that the optimal veto would be longer than at JUNO but shorter than 2 seconds, nonetheless as a result of the low muon rate KamLAND does not need a precise optimization of the veto time. On the other hand, at JUNO the conflicting constraints imposed by the large background, which requires a long veto, and the statistical fluctuations in the hierarchy-sensitive region of the spectrum around $3 \mathrm{MeV}$, which require a short veto, leave a small window of acceptable veto strategies for the hierarchy determination at JUNO.

We have reduced the number of independent parameters from 3 to 2 , only $E_{c}$ and $x$ need to be optimized. The problem is now identical to that of the full detector veto treated above but now $x$ plays the role that was played by $t_{f}$, or strictly speaking by $1-e^{-t_{f} / t_{l}}$. Fixing the rejection efficiency $\mathcal{E}_{c}$, eq. (3.7) gives the showering energy threshold $E_{c}$

$$
E_{c}\left(\mathcal{E}_{c}, x\right)=l^{-1}\left(\frac{\mathcal{E}_{c}}{x}\right)
$$

Substituting this value of $E_{c}\left(\mathcal{E}_{c}, x\right)$ into (3.9) one obtains $t_{l}\left(\mathcal{E}_{c}, x\right)$. Thus, as in the case of a full detector veto (3.4), for each rejection efficiency $\mathcal{E}_{c}$ one determines the optimal $x\left(\mathcal{E}_{c}\right)$ by demanding that it extremizes $t_{l}\left(\mathcal{E}_{c}, x\right)$ with $\mathcal{E}_{c}$ held fixed. This optimum is shown in figure 8.

Once $x\left(\mathcal{E}_{c}\right)$ has been determined, eq. (3.11) yields the threshold showering energy $E_{c}$ for the cylindrical veto, shown in figure 9 . For an efficiency $\mathcal{E}_{c}$ near $99 \%$ this threshold should be quite low, near $1 \mathrm{GeV}$. However a more modest efficiency of $90 \%$ requires a threshold of $3 \mathrm{GeV}$, equal to the threshold used by KamLAND for its full detector veto.

Combining figure 8 for $x$ as a function of $\mathcal{E}_{c}$ with figure 7 which provides $r_{c}$ and $t_{c}$ as a function of $x$ one finds the cylindrical veto parameters $r_{c}$ and $t_{c}$ as a function of the efficiency $\mathcal{E}_{c}$. These are shown in figure 10. Finally, as the fractional live time is now quite close to 


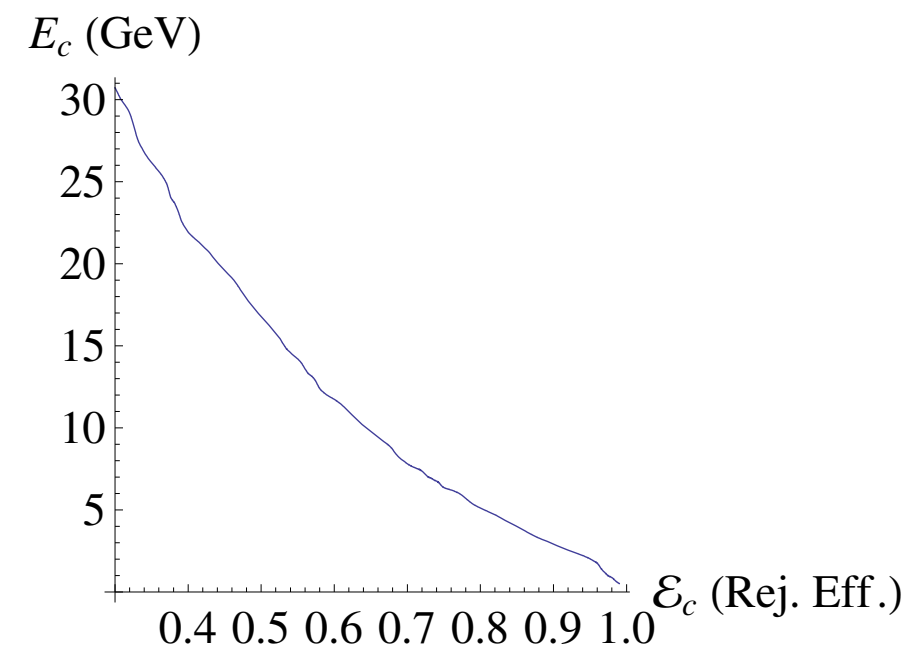

Figure 9. The optimal showering energy threshold $E_{c}$, as a function of the efficiency $\mathcal{E}_{c}$ of a cylindrical veto.
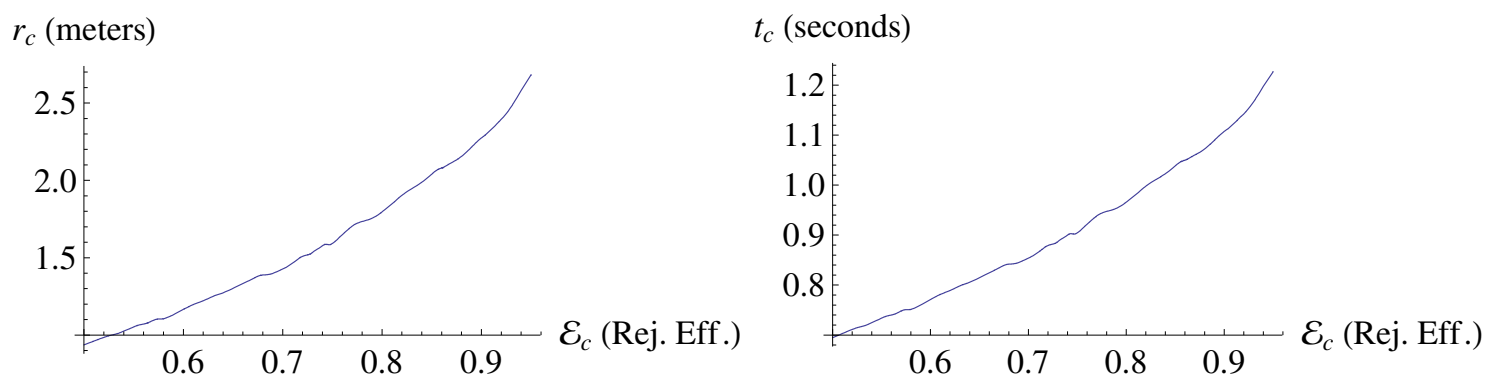

Figure 10. The optimal cylindrical veto radius $r_{c}$ and time $t_{c}$ for a fixed rejection effiency $\mathcal{E}_{c}$.

unity, we plot the fractional dead time $1-t_{l}$ in figure 11. This is one of the main results of our study. Note that a rejection efficiency of $90 \%$ can be obtained while losing only $3 \%$ of the IBD signal, while a $95 \%$ efficiency costs $10 \%$ of the signal. However the commonly quoted goal of $99 \%$ background rejection would require a loss of $35 \%$ of the IBD events, and this under the assumption of perfect tracking which is quite unlikely for $99 \%$ of muons.

\section{Optimal veto for each science goal}

In section 3 for each kind of veto, full detector or cylindrical, we obtained the maximum fractional live time $t_{l}$ possible for each ${ }^{9} \mathrm{Li}$ rejection efficiency $\mathcal{E}_{f}$ or $\mathcal{E}_{c}$ and we found the corresponding veto parameters $E_{f}, E_{c}, r_{c}, t_{f}$ and $t_{c}$ which are functions of the rejection efficiencies.

In this section we will assume that the muon tracking is perfect, leaving the general case to section 5 . Therefore we will only be interested in cylindrical vetoes. The cylindrical veto is then fully described by a single parameter, the efficiency $\mathcal{E}_{c}$. The showering energy threshold $E_{c}\left(\mathcal{E}_{c}\right)$, veto cylinder radius $r_{c}\left(\mathcal{E}_{c}\right)$ and veto duration $t_{c}\left(\mathcal{E}_{c}\right)$ may be read from figures 9 and 10 while the corresponding fractional dead time was reported in figure 11. 


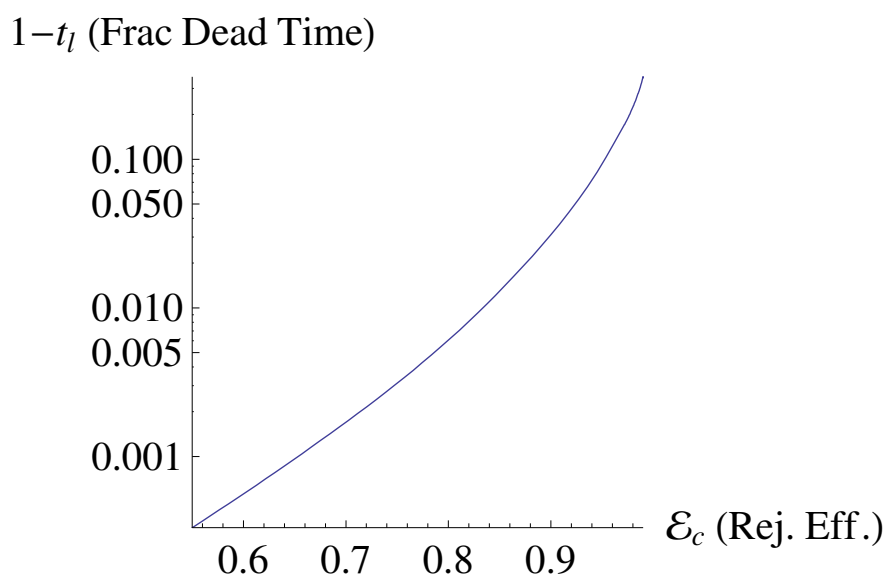

Figure 11. The fractional volume-weighted dead time $1-t_{l}$, as a function of the efficiency $\mathcal{E}_{c}$ of a cylindrical veto.

But what is the optimal value of $\mathcal{E}_{c}$ ? This depends on the science goal considered. For each science goal, we have calculated a statistic characterizing the goal. This statistic depends on the total live time of the experiment and on the total background during that live time. We have assumed that the experiment runs for 6 years, and so the live time will just be $6 t_{l}$ years. As $t_{l}$ is known as a function of $\mathcal{E}_{c}$ (figure 11), one may then obtain the statistic as a function of $\mathcal{E}_{c}$ alone and then choose the $\mathcal{E}_{c}$ which optimizes the statistic, optimizing the veto strategy for that science goal. As the vetoes are not hardwired, there is no problem using a different veto strategy for each science goal.

\subsection{Neutrino mass hierarchy}

In this subsection we will optimize the veto strategy for the determination of the neutrino mass hierarchy at JUNO. For this purpose, we have computed the statistic $\Delta \chi^{2}$ which is equal to the difference between the $\chi^{2}$ value of a best fit of a given spectrum to the normal and inverted hierarchies

$$
\Delta \chi^{2}=\chi^{2}(\text { normal })-\chi^{2} \text { (inverted) }
$$

In figure 12 we display $\Delta \chi^{2}$ computed for an Asimov data set generated using the inverted hierarchy, using 6 years of run time, which throughout this paper we assume corresponds to $1.35 \times 10^{5}$ inverse $\beta$ decay events.

Note that $\Delta \chi^{2}$ is not that of Wilks' theorem, which is defined to be the difference between a best $\chi^{2}$ fit to a null hypothesis and that to a best fit of a continuous family of parameters of which the null hypothesis is one choice. Indeed, the choice of hierarchies is not even continuous. For example, the quantity $\Delta \chi^{2}$ defined in eq. (4.1) is Gaussiandistributed [11-13] whereas that in Wilks' theorem is distributed following a $\chi^{2}$ distribution. Therefore this $\Delta \chi^{2}$ is not the square of the number of $\sigma$ of confidence in a null hypothesis. However, in a Bayesian framework in which one assigns a symmetric prior to the two hierar- 


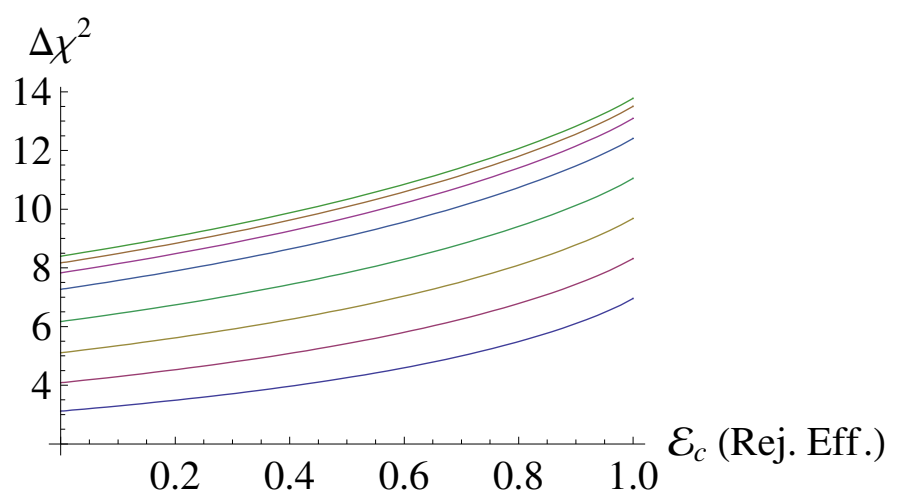

Figure 12. The $\Delta \chi^{2}$ with which the neutrino mass hierarchy may be determined by JUNO in 6 years with a fraction $t_{l}$ of live time and ${ }^{9} \mathrm{Li}$ rejection efficiency $\mathcal{E}_{c}$. For the curves from top to bottom the fractions $t_{l}$ are $1.0,0.98,0.95,0.9,0.8,0.7,0.6$ and 0.5 . It is assumed that the nonlinear energy response of the detector is known perfectly.

chies, this $\Delta \chi^{2}$ is simply related to the sensitivity $s$ to the hierarchy via the relation [11-13]

$$
s=\frac{1}{1+e^{-\Delta \chi^{2} / 2}} .
$$

If this sensitivity is converted into a number of $\sigma$ using the error function, one finds a reduction of about $0.5 \sigma$ as compared with the naive square root formula.

The detector energy resolution, number of events, etc. are as in ref. [14] and conform to the experimental goals. However we assume that the nonlinear energy response of the detector is perfectly known. In fact the experimental goal is a $1 \%$ precision on the nonlinear energy response, which has a large effect on $\Delta \chi^{2}$ as has been foreseen in ref. [15] and demonstrated in ref. [16]. However the precise effect on $\Delta \chi^{2}$ depends heavily on additional assumptions, such as the shape of the nonlinearity. It may be possibile to estimate the nonlinearity by using the known general features of the spectrum [1], however such a strategy may be ineffective or even problematic if the reactor neutrino spectrum has unexpected features, such as the $5 \mathrm{MeV}$ bump first reported by RENO [17, 18] and later confirmed by Double Chooz [19] and Daya Bay [20]. Therefore the inclusion of the unknown nonlinear energy response in this study would introduce uncertainties which would obfuscate the effect of the spallation backgrounds.

The optimization of the rejection efficiency $\mathcal{E}_{c}$ is now quite easy. One need only substitute figure 11 , which gives $t_{l}$ as a function of $\mathcal{E}_{c}$, into figure 12 which gives $\Delta \chi^{2}$ as a function of $t_{l}$ and $\mathcal{E}_{c}$ to obtain $\Delta \chi^{2}$ as a function of $\mathcal{E}_{c}$ alone, shown in figure 13. One can see that, in contrast with the common claim that the optimal rejection efficiency at JUNO will be at least $99 \%$, the optimal rejection efficiency is in fact only $90 \%$ when the tracking is perfect. The drop in $\Delta \chi^{2}$ for $\mathcal{E}_{c}$ above $90 \%$ is one of the main results of our study. In figure 11 one can observe that the optimal rejection efficiency of $90 \%$ yields a dead time of about $3 \%$, which increases to nearly $50 \%$ for a rejection efficiency of $99 \%$. Thus the curves in figure 12 correspond to the optimal dead times for the drop on the right hand side of 


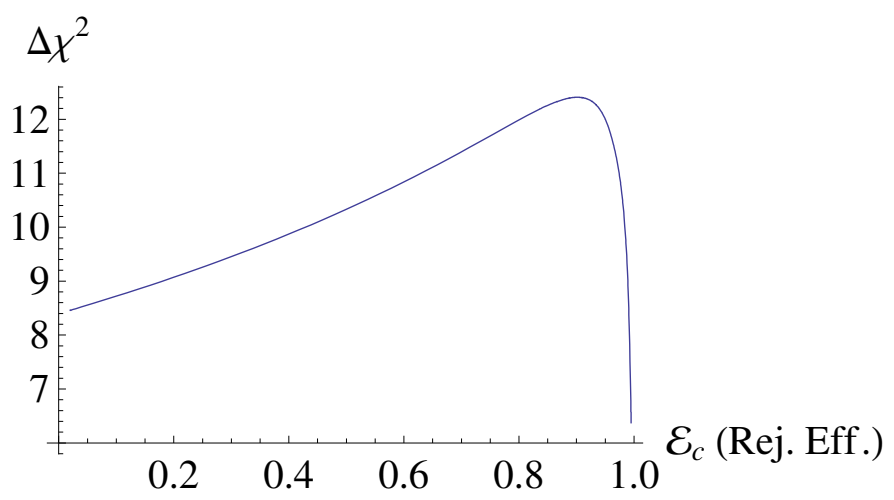

Figure 13. The $\Delta \chi^{2}$ with which the neutrino mass hierarchy may be determined by JUNO in 6 years as a function of the rejection efficiency $\mathcal{E}_{c}$. The optimal rejection efficiency is $90 \%$.

figure 13. Using figures $8,9,10$ and 11 with $\mathcal{E}_{c}=0.9$, one may obtain the corresponding optimal veto parameters, which are summarized in table 1.

Despite the relatively large contamination, in the case of perfect tracking the ${ }^{9} \mathrm{Li}$ background only reduces $\Delta \chi^{2}$ from 13.8 (with no background) to 12.4 (with the full background). This is our main result.

If Taishan reactors 3 and 4 are not built, then the reactor neutrino signal at JUNO will be reduced by about $26 \%$. This can be incorporated into the present analysis with a $26 \%$ reduction of the live time $t_{l}$ in figure 12 , resulting in a $20-25 \%$ reduction in $\Delta \chi^{2}$.

\subsection{Uncertainty in the ${ }^{9} \mathrm{Li}$ spectrum}

The small reduction in $\Delta \chi^{2}$ is a result of the fact that the shape of the ${ }^{9} \mathrm{Li}$ decay background is known quite well, and so can be reliably subtracted. What is the shape? First, recall that we are only interested in decays producing neutrons

$$
{ }^{9} \mathrm{Li} \rightarrow{ }^{9} \mathrm{Be}^{*}+\mathrm{e}^{-}+\bar{\nu}_{\mathrm{e}} \rightarrow \mathrm{n}+2 \alpha+\mathrm{e}^{-}+\bar{\nu}_{\mathrm{e}}
$$

where ${ }^{9} \mathrm{Be}^{*}$ is an excited state. The $\bar{\nu}_{e}$ is not observed. The spectrum of the $e^{-}$is known quite precisely from Fermi's theory of $\beta$ decay. It extends up to about $11 \mathrm{MeV}$ and overlaps with the entire reactor neutrino prompt energy spectrum. Indeed the scintillator detector cannot distinguish these $e^{-}$from the $e^{+}$produced by the inverse $\beta$ capture of reactor neutrinos. The neutron and $\alpha$ particles are produced essentially immediately after the $\beta$ decay.

However, as $90 \%$ of neutron-emitting decays begin with a $\beta$ decay to the low energy (less than $3 \mathrm{MeV}$ ) excited states of ${ }^{9} \mathrm{Be}$ [21], little energy is available for the neutron and $\alpha$ particles. As a result, these particles have kinetic energies in general well below $2 \mathrm{MeV}$, often below $1 \mathrm{MeV}$. In this regime their scintillator quenching factors are large. The $\alpha$ particle quenching factor is more than 10 times that of the electron, reducing its contribution to the scintillation light by a factor of more than 10 so that its contribution to the observed energy negligible. The neutron's quenching factor is more than 5 times that of the electron, so its contribution is small but not negligible. The neutron's energy begins to arrive in the scintillator as it slows. The neutrons slows as it collides with particles in the scintillator. 
The time between such collisions is about $5 \mathrm{~ns}$ and about half of the collisions, those with free protons, reduce the neutron's energy by about one half. Therefore the neutron loses most of its energy in about 10 ns.

While one might think that the PMTs could then distinguish the neutron's energy from that of the electrons using the $10 \mathrm{~ns}$ time difference and so veto the ${ }^{9} \mathrm{Li}$ events, this is not the case. The main problem is the time-lag before the scintillator scintillates, which ranges from 10 s to 100 s of ns. Another problem is that JUNO is about the same size as the mean free path of the ultraviolet photons created by the wave shifters. As a result, a significant portion of the photons created by the electron bounce inside of the scintillator before arriving at the PMTs, and so arrive 10s of nanoseconds late. These late photons from the electron cannot be distinguished from the photons from the neutron slowing, and so one only observes the total energy. However, the neutron energy was already 5-10 times smaller than the electron energy and the quenching factor reduces its contribution to the visible energy by an additional factor of 5 , thus the neutron contributes only about $5 \%$ of the visible energy. Now the neutron spectrum, although it has been measured by many experiments [22-24], is not known as precisely as the electron spectrum. Nonetheless, the fact that it contributes only $5 \%$ of the visible energy means that the uncertainty in the neutron spectrum yields a very small uncertainty in the ${ }^{9} \mathrm{Li}$ spectrum. This is why the ${ }^{9} \mathrm{Li}$ spectrum is so well known, which allows the presence of a large ${ }^{9} \mathrm{Li}$ contaminant in the IBD event sample to have such a small effect on the sensitivity to the hierarchy. JUNO itself will provide a very precise measurement of the quenching factor weighted spectrum, actually of a weighted combination of the ${ }^{9} \mathrm{Li}$ and ${ }^{8} \mathrm{He}$ spectra, which will only decrease this uncertainty.

In fact, the biggest uncertainty in the ${ }^{9} \mathrm{Li}$ spectrum may arise from the veto efficiency $\mathcal{E}_{c}$, which depends upon the the precision of the reconstruction of the muon track and the radial distribution $f(R)$ of the ${ }^{9} \mathrm{Li}$ which is not well known, although JUNO will measure it better than it has ever been measured before. Unlike the previous uncertainty, this does not affect the shape of the observed energy spectrum, but only the overall normalization. The precision of these estimates are quite difficult to forecast. Therefore in this note we simply assume that the shape of the ${ }^{9} \mathrm{Li}$ spectrum is known perfectly and we include a $1 \%$ uncertainty in its overall normalization.

\subsection{Mixing angle $\theta_{12}$}

In this subsection we apply the same analysis to the determination of $\theta_{12}$ as that used for the determination of the hierarchy in subsection 4.1. We will choose the veto strategy to minimize $\sigma\left(\sin ^{2}\left(2 \theta_{12}\right)\right)$, the precision with which $\sin ^{2}\left(2 \theta_{12}\right)$ can be determined. There are various calculations of this uncertainty in the literature. The earliest [25, 26] do not consider the uncertainty in the shape of the reactor neutrino spectrum. Later calculations, such as ref. [27], do consider this uncertainty but use the theoretical lower bounds on the uncertainty given in ref. [28]. However, it is now known that the lower bound on this uncertainty is far from saturated, indeed it would imply that the $5 \mathrm{MeV}$ bump $[17,18,20]$ is excluded at $4 \sigma$.

This motivates us to use the more recent determination of $\sigma\left(\sin ^{2}\left(2 \theta_{12}\right)\right)$ from ref. [29], shown in figure 14, which uses the observational uncertainty on the reactor neutrino flux 


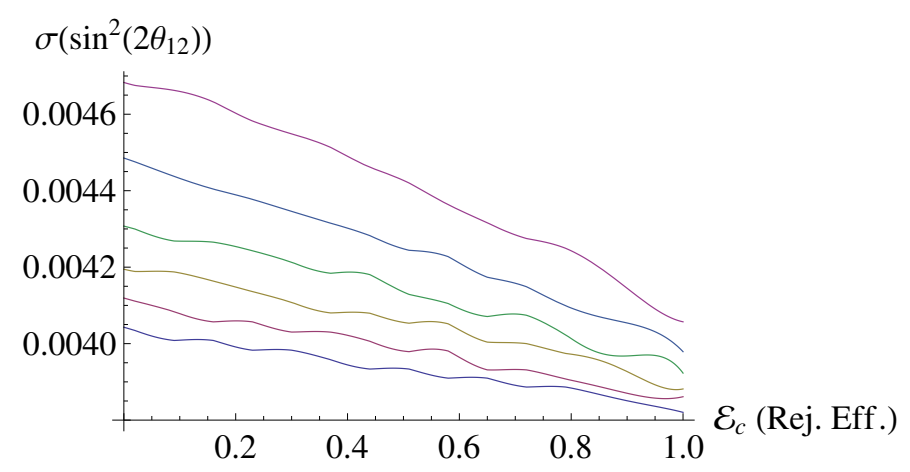

Figure 14. The $1 \sigma$ uncertainty with which $\sin ^{2}\left(2 \theta_{12}\right)$ can be measured at JUNO in 6 years with a fraction $t_{l}$ of live time and ${ }^{9} \mathrm{Li}$ rejection efficiency $\mathcal{E}_{c}$, from ref. [29]. For the curves from bottom to top the fractions $t_{l}$ are $1.0,0.9,0.8,0.7,0.6$ and 0.5 . The main contribution to the uncertainty arises from the uncertainty in the reactor spectrum measured by Daya Bay [20].

from Daya Bay [20]. In addition, this is the only study which yields $\sigma\left(\sin ^{2}\left(2 \theta_{12}\right)\right)$ as a function of the dead time and the ${ }^{9} \mathrm{Li}$ veto efficiency, which is necessary to optimize the vetoes.

The most obvious distinction between figure 14 and the corresponding figure for the mass hierarchy, figure 12, is that in the former the experimental performance hardly depends upon the rejection efficiency and the dead time in the range plotted. This is because the precision of a determination of $\theta_{12}$ is dominated by systematic errors, the uncertainty in the reactor spectrum, unlike the determination of the hierarchy. Of course, if we included the uncertainty in the detector's energy response then then the systematic errors would play a more important role in the determination of the hierarchy. However, so long as this response can be understood well enough to achieve a reasonable determination of the hierarchy, it will play a subdominant role.

As in the case of the hierarchy, we now substitute figure 11, which gives $t_{l}$ as a function of $\mathcal{E}_{c}$, into figure 14 which gives $\sigma\left(\sin ^{2}\left(2 \theta_{12}\right)\right)$ as a function of $t_{l}$ and $\mathcal{E}_{c}$ to obtain $\sigma\left(\sin ^{2}\left(2 \theta_{12}\right)\right)$ as a function of $\mathcal{E}_{c}$ alone, shown in figure 15 . Note that the optimal rejection efficiency is now $93 \%$, as compared with $90 \%$ for the mass hierarchy and the precision $\sigma\left(\sin ^{2}\left(2 \theta_{12}\right)\right)$ is 0.0038 or $0.45 \%$, as compared with $0.44 \%$ with no background at all. As summarized in table 1 this means that the optimal veto strategy for the determination of $\theta_{12}$ should have $6 \%$ dead time, as compared with only $3 \%$ for the neutrino mass hierarchy. The reason for this is that the error in $\theta_{12}$ is dominated by the systematic error in the reactor spectrum, not by statistical fluctuations like the measurement of the hierarchy. Therefore a larger dead time in the $\theta_{12}$ analysis has less effect on the precision of the measurement than it would in a determination of the hierarchy.

Physically this is a consequence of the fact that $\theta_{12}$ is determined by a broad feature in the reactor neutrino spectrum, essentially the difference between the neutrino flux at intermediate energies at the solar oscillation maximum and that at higher and lower energies. Being a broad feature it is insensitive to the energy resolution of the detector and more importantly it involves essentially the whole sample of neutrinos. Thus the statistical uncertainty on $\theta_{12}$ is extremely small. The determination of the hierarchy is quite different. 


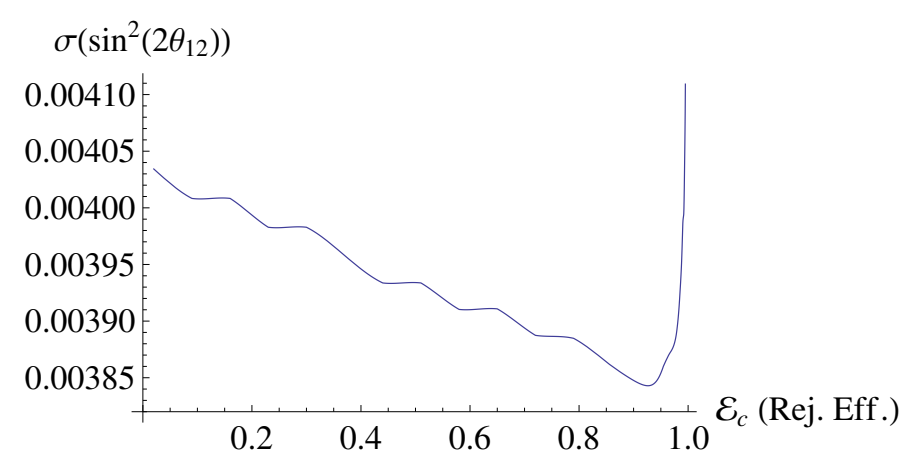

Figure 15. The $1 \sigma$ uncertainty with which $\sin ^{2}\left(2 \theta_{12}\right)$ can be measured by JUNO in 6 years as a function of the rejection efficiency $\mathcal{E}_{c}$. The optimal rejection efficiency is $93 \%$, higher than for the hierarchy because the uncertainty is dominated by a systematic error, the uncertainty in the reactor neutrino spectrum, which is taken to be as in figure 14 .

\begin{tabular}{|c|l|l|l|l|l|l|l|}
\hline Science Goal & Statistic & $\mathcal{E}_{c}$ & $t_{l}$ & $E_{c}$ & $x$ & $t_{c}$ & $r_{c}$ \\
\hline Mass hierarchy & $\Delta \chi^{2}=12.4$ & $90 \%$ & $97 \%$ & $2.9 \mathrm{GeV}$ & $95 \%$ & $1.1 \mathrm{sec}$ & $2.3 \mathrm{~m}$ \\
\hline Measure $\theta_{12}$ & $\frac{\sigma\left(\sin ^{2}\left(2 \theta_{12}\right)\right)}{\sin ^{2}\left(2 \theta_{12}\right)}=0.45 \%$ & $93 \%$ & $94 \%$ & $2.4 \mathrm{GeV}$ & $96 \%$ & $1.2 \mathrm{sec}$ & $2.5 \mathrm{~m}$ \\
\hline
\end{tabular}

Table 1. The optimal cylindrical veto parameters for the determination of the mass hierarchy and $\theta_{12}$ assuming perfect tracking.

It comes from comparing $\Delta M_{e e}^{2}$ [30] measured at intermediate and high energies with the position of the 15 th, 16 th and 17 th peaks at low energies [26, 31]. These low energy peaks contain few events, especially when one considers the effect of the finite energy resolution and interference from neutrinos coming from reactors at distinct baslines [31] at JUNO. Therefore statistical uncertainty is very important in the determination of the hierarchy, which drives the preference for a veto strategy with little dead time.

\section{$5 \quad$ Imperfect tracking}

In this section we will consider the effects of imperfect tracking of muons. We will make the poor approximation that the probability of tracking a muon is independent of its showering energy. Up to this point the generalization to muon bundles has been straightforward, it only required modifying the functions $m(E)$ and $l(E)$ by including the weighted sum of bundles. However if one introduces bundles, then one either needs to introduce new parameters describing how well the bundles are tracked, or else make the further crude approximation that the tracking of each muon is independent of whether it arrives in a single or multimuon event. In the latter case, the treatment below remains applicable, one need only remember that $\alpha$ and $\beta$, which will be defined momentarily, are fractions of muons, not of muon bundles.

With these caveats, the tracking is described by just two parameters, $\alpha$ and $\beta$, which, as is summarized in table 2 are the fraction of muons for which the tracking fails but is believed to succeed and the fraction of muons for which the experimenter believes, 


\begin{tabular}{|c|l|}
\hline Variable & Tracking \\
\hline $1-\alpha-\beta$ & Muon well tracked \\
\hline$\alpha$ & Muon poorly tracked but believed to be well tracked \\
\hline$\beta$ & Muon believed to be poorly tracked \\
\hline
\end{tabular}

Table 2. The tracking of muons is characterized by the parameters $\alpha$ and $\beta$, assumed to be independent of the showering energy.

correctly or incorrectly, that the tracking has failed. Our veto strategy then demands that full detector vetoes be applied to a fraction $\beta$ of all muons. In the case of the $\alpha$ muons a cylindrical veto is applied, but as it is quite unlikely that this cylinder coincides accidentally with the muon track, we will simply assume that the veto does not increase the rejection efficiency. The fraction of muons for which the tracking is successful, and the experimenter knows that the tracking is successful, is $1-\alpha-\beta$.

For simplicity we will make the crude approximation that the tracks of well-tracked muons are determined precisely. In fact, one expects a precision of only 1-2 meters. However, this precision depends on the configuration of the top veto, which is not yet fixed, and so cannot be estimated reliably at this time. The result of the uncertainty in the muon track position would be to increase the optimal $r_{c}$ by 1 meter or more and indeed it may be worthwhile to consider a cylinder which gets wider along the muon's trajectory. As a consequence, the total dead time will be higher than that estimated below and the rejection efficiency lower.

The resulting fractional live time is

$$
t_{l}\left(E_{c}, r_{c}, x\right)=\exp \left(-(1-\beta) \frac{\pi d\left(E_{c}\right) r_{c}^{2}}{V} t_{c} m\left(E_{c}\right)-\beta t_{f} m\left(E_{f}\right)\right) .
$$

The veto strategy now depends on two parameters, the rejection efficiencies $\mathcal{E}_{f}$ and $\mathcal{E}_{c}$ for the full detector and the cylindrical vetoes. On the other hand, the science goals only depend upon $t_{l}$ and the total rejection efficiency

$$
\mathcal{E}=(1-\alpha-\beta) \mathcal{E}_{c}+\beta \mathcal{E}_{f}
$$

where we have used the assumption that in the case of bad tracking, cylindrical vetoes do not reject any background. Therefore the optimization of the veto strategy will consist of two steps. First, for each $\mathcal{E}$, we will find $\mathcal{E}_{c}$ and $\mathcal{E}_{f}$ so as to maximize $t_{l}$. Next, in the following two subsections, we will choose $\mathcal{E}$ for each science goal so that the corresponding statistic is optimized.

The total live time $t_{l}$ in eq. (5.1) has one very useful feature. It is monotonic in both the live times $t_{l}$ in eq. (3.3) and in eq. (3.9) of the full detector and cylindrical vetoes, in fact it is just a product of a positive powers of the two live times

$$
t_{l}=\left[t_{l} \text { (full detector) }\right]^{\beta}\left[t_{l} \text { (cylindrical) }\right]^{1-\beta} .
$$

Therefore the $t_{f}\left(\mathcal{E}_{f}\right), E_{f}\left(\mathcal{E}_{f}\right), t_{c}\left(\mathcal{E}_{c}\right), r_{c}\left(\mathcal{E}_{c}\right)$ and $E_{c}\left(\mathcal{E}_{c}\right)$ that minimized the $t_{l}$ of eqs. (3.3) and (3.9) for each individual veto in section 3 also minimize the total $t_{l}$ of eq. (5.1). This 

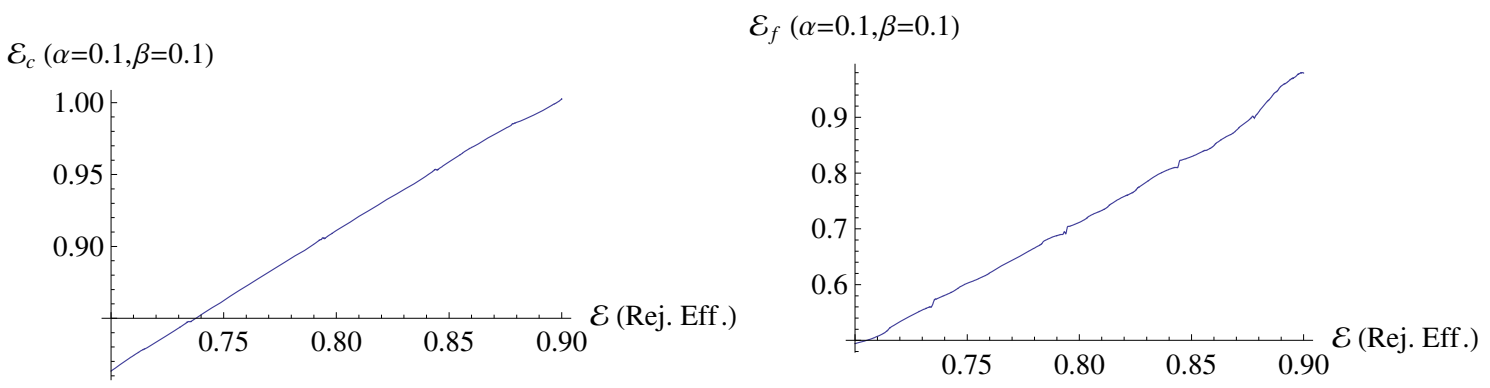

Figure 16. The optimal cylindrical and full detector veto efficiencies $\mathcal{E}_{c}$ and $\mathcal{E}_{f}$ as a function of the total veto efficiency $\mathcal{E}$. Here $\alpha=\beta=0.1$ which means that $80 \%$ of muons are well tracked but $90 \%$ are believed to be well tracked.

greatly simplifies our analysis, as it means that we can continue to use the functions $t_{f}\left(\mathcal{E}_{f}\right)$, $E_{f}\left(\mathcal{E}_{f}\right), t_{c}\left(\mathcal{E}_{c}\right), r_{c}\left(\mathcal{E}_{c}\right)$ and $E_{c}\left(\mathcal{E}_{c}\right)$ that we found assuming perfect tracking.

Our goal in this section is to obtain $\mathcal{E}_{f}(\mathcal{E})$ and $\mathcal{E}_{c}(\mathcal{E})$. When $\beta \neq 0$, the former is easily obtained from eq. (5.2)

$$
\mathcal{E}_{f}(\mathcal{E})=\frac{\mathcal{E}-(1-\alpha-\beta) \mathcal{E}_{c}(\mathcal{E})}{\beta} .
$$

Thus we need only substitute $\mathcal{E}_{f}(\mathcal{E})$ from eq. (5.4) into the expression (5.1) for the total live time and then choose $\mathcal{E}_{c}(\mathcal{E})$ so as to minimize the total live time.

However the function $\mathcal{E}_{c}(\mathcal{E})$ also depends on the parameters $\alpha$ and $\beta$ and so it is not easy to display in a figure. While we have done this optimization for every $\alpha$ and $\beta$, in this subsection we display, in figure 16, the optimal cylindrical and full detector veto efficiencies only for $\alpha=\beta=0.1$. This corresponds to the case in which the tracking is successful for $80 \%$ of muons but the experimenter believes that it has been successful for $90 \%$ of muons, therefore the cylindrical veto is used $90 \%$ of the time. Note that in general the optimal full detector rejection efficiency is lower than the rejection efficiency for the cylindrical vetoes. This is because full detector vetoes lead to a much larger dead time. The maximum possible rejection efficiency $\mathcal{E}$ is $1-\alpha=90 \%$, as a fraction $\alpha=10 \%$ of the muons are poorly tracked unbeknownst to the experimentalist and so the cylindrical veto covers the wrong region.

The corresponding fractional dead time $1-t_{l}$ is plotted in figure 17. Although only $80 \%$ of the muons are successfully tracked, the fact that half of the poorly tracked muons are known to be poorly tracked nonetheless allows a rejection efficiency of $85 \%$ with a dead time of $13 \%$, comparable to KamLAND.

\subsection{The neutrino mass hierarchy}

Given the full detector and cylindrical rejection efficiencies $\mathcal{E}_{f}$ and $\mathcal{E}_{c}$, one can determine the $\Delta \chi^{2}$ with which one expects the true hierarchy to be favored for any tracking parameters $\alpha$ and $\beta$. In the case $\alpha=\beta=0.1$, this is plotted in figure 18 for some choices of rejection efficiencies.

The optimization of the veto for the neutrino mass hierarchy is now quite simple. Only one independent parameter remains to be fixed, the total rejection efficiency $\mathcal{E}$. Similarly to the case with perfect tracking, the sensitivity to the hierarchy as a function of $\mathcal{E}$ is 


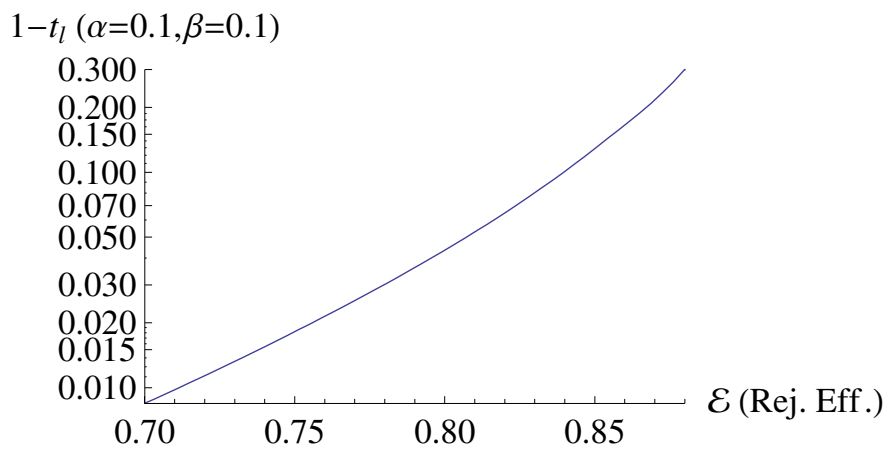

Figure 17. The fractional dead time which is required to obtain a total rejection efficiency $\mathcal{E}$ with $\alpha=\beta=0.1$.

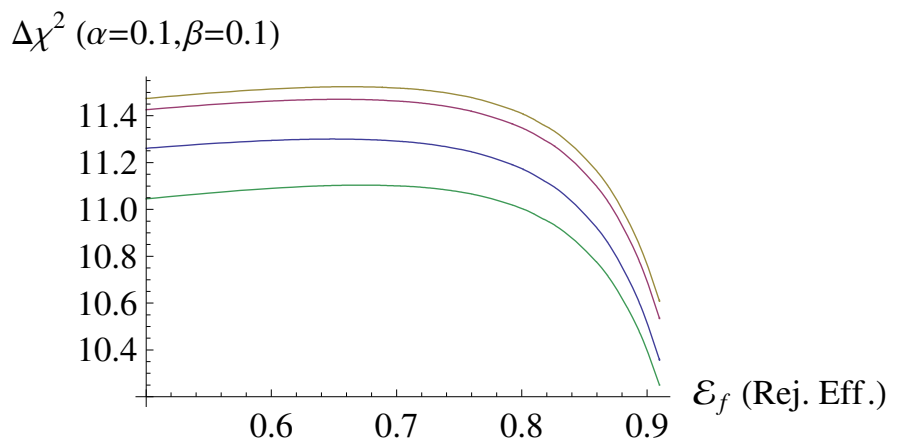

Figure 18. The statistic $\Delta \chi^{2}$ which characterizes the sensitivity to the neutrino mass hierarchy as a function of the full detector rejection efficiency $\mathcal{E}_{f}$ with $\alpha=\beta=0.1$. From top to bottom, the curves correspond to cylindrical rejection $\mathcal{E}_{c}$ effiiciencies of $90 \%, 85 \%, 80 \%$ and $99 \%$. Note that the highest value of $\Delta \chi^{2}$ visible, lying on the $\mathcal{E}_{c}=90 \%$ curve, is quite close to the absolute optimum of $\mathcal{E}_{c}=89 \%$ and $\mathcal{E}_{f}=66 \%$ given in table 3 .

obtained by restricting figure 12 to the pairs $\left(\mathcal{E}, t_{l}\right)$ on the curve in figure 17 , corresponding to the maximal live time achievable for each rejection efficiency. This yields figure 19. Here one can see that with $\alpha=\beta=0.1$, the optimal rejection efficiency is $78 \%$ and it yields a $\Delta \chi^{2}$ of 11.5 , as compared with 13.8 with no background and 12.4 with perfect tracking. The corresponding optimal veto parameters are summarized in table 3.

For general tracking efficiency parameters $\alpha$ and $\beta$, one can follow this same strategy to optimize the total rejection efficiency $\mathcal{E}$. The results are shown in figure 20 together with the corresponding fractional live times obtained using figure 17. The corresponding statistic $\Delta \chi^{2}$ is presented in figure 21. This is one of our main results. It shows the dependence of the sensitivity to the hierarchy on the tracking. It is evident that $\alpha$ has a greater impact upon $\Delta \chi^{2}$ than $\beta$, reflecting the fact that if it is known that the tracking has failed (as occurs for a fraction $\beta$ of muons) then one can nonetheless perform a full detector veto which increases the sensitivity to the hierarchy. One can see that the failure to track $10 \%$ of muons does not greatly reduce the sensitivity to the hierarchy. However, muon bundles are responsible for about $30 \%$ of muon events, and so the ability to track bundles 


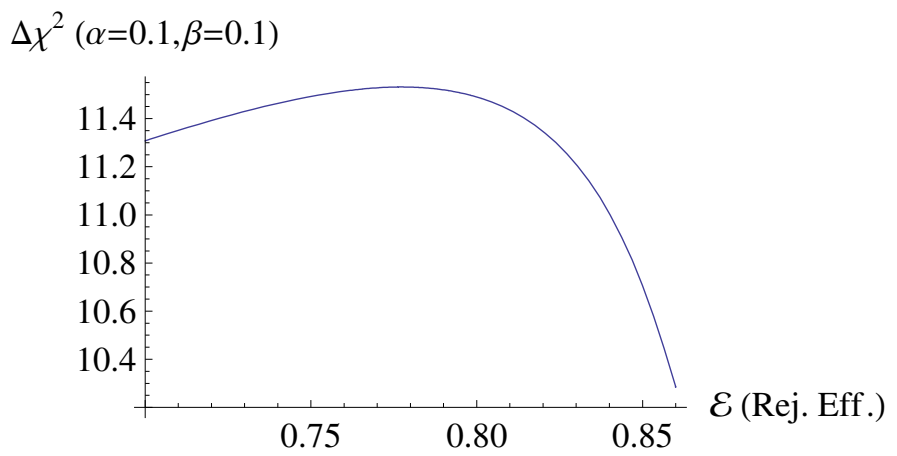

Figure 19. The statistic $\Delta \chi^{2}$ which characterizes the sensitivity to the neutrino mass hierarchy as a function of the total rejection efficiency $\mathcal{E}$ with $\alpha=\beta=0.1$.
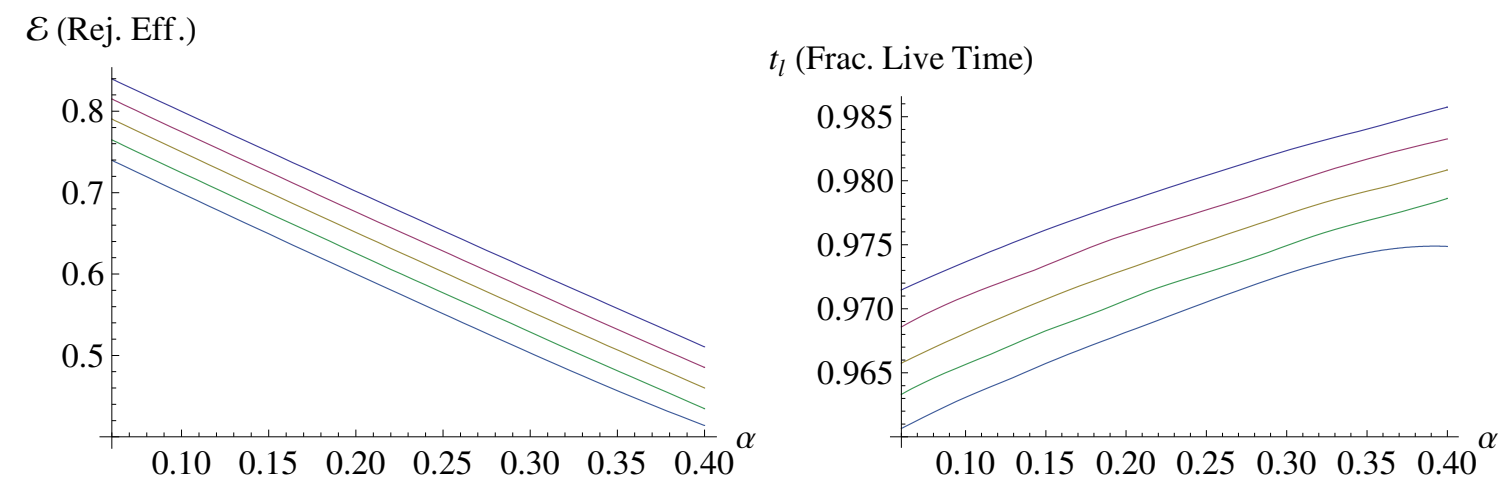

Figure 20. The optimal veto efficiency and total fractional live time for the determination of the mass hierarchy as a function of the tracking efficiency parameters $\alpha$ and $\beta$. The curves, from top to bottom, correspond to $\beta=0,0.1,0.2,0.3$ and 0.4 .

does have a nonnegligible effect on the sensitivity. KamLAND has several classifications of how reliably a given muon was tracked [32]. A tighter reliability threshold corresponds to a larger value of $\beta$ and a smaller value of $\alpha$. The results of our study can be used to optimize this reliability threshold.

We have now optimized the veto strategy, for the determination of the neutrino mass hierarchy, as a function of $\alpha$ and $\beta$. For completeness, we will now describe this optimal veto. In figure 22 one can find the individual veto efficiencies for the cylindrical and full detector vetoes. Surprisingly, the individual efficiencies are quite independent of the tracking, one always wants a rejection efficiency in the mid to high eighties for the cylindrical veto and in the mid sixties for the full detector veto. As these individual efficiencies give the veto parameters, using the results of section 3 , this implies that the optimal veto parameters themselves are quite insensitive to the tracking abilities of the detector. The optimal parameters are shown in figures 23 and 24 for the cylindrical and full detector vetoes respectively.

\subsection{Measuring $\theta_{12}$}

The measurement of $\theta_{12}$ can be analyzed similarly to the hierarchy determination. The precision $\sigma\left(\sin ^{2}\left(2 \theta_{12}\right)\right)$ as a function of $\mathcal{E}$ is obtained by restricting figure 14 to the pairs 


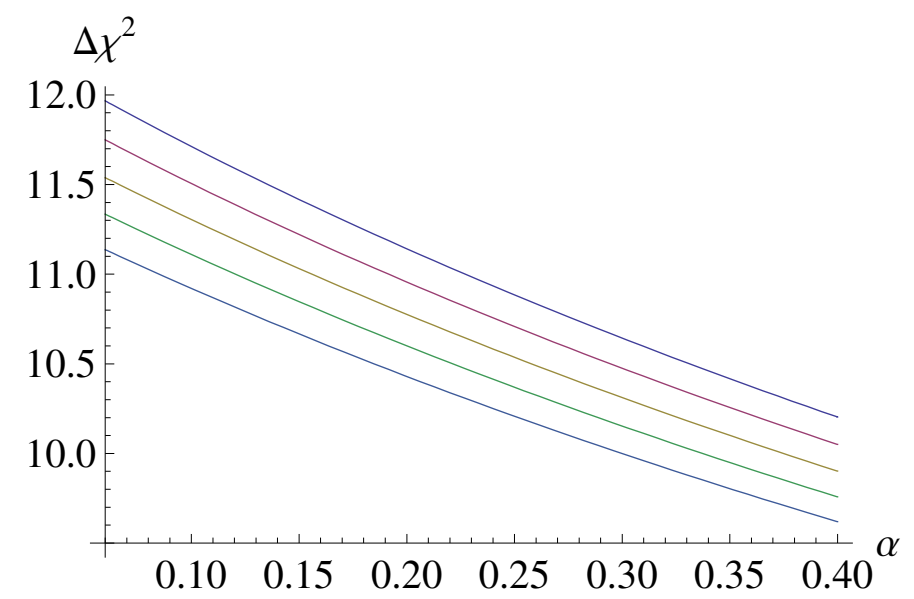

Figure 21. The statistic $\Delta \chi^{2}$ which characterizes the sensitivity to the neutrino mass hierarchy as a function of the fraction $\beta$ of muons which are known to be poorly tracked and the fraction $\alpha$ of muons which appear to be well tracked but in fact are poorly tracked. The curves, from top to bottom, correspond to $\beta=0,0.1,0.2,0.3$ and 0.4 .
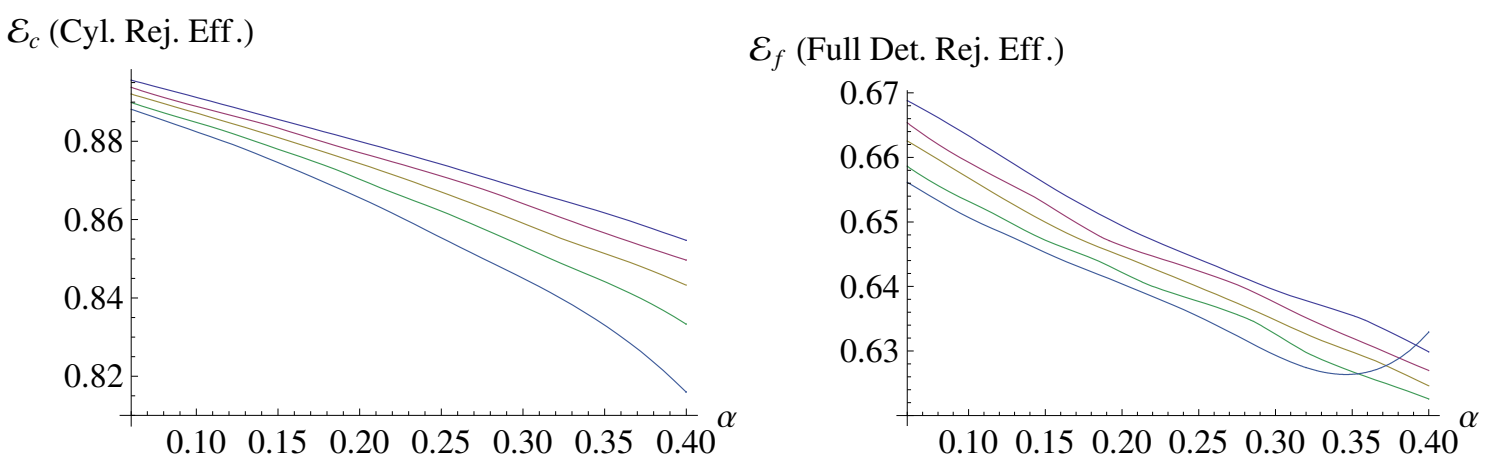

Figure 22. The optimal cylindrical and full detector veto efficiencies as a function of the tracking efficiency parameters $\alpha$ and $\beta$. The curves, from top to bottom, correspond to $\beta=0,0.1,0.2,0.3$ and 0.4.
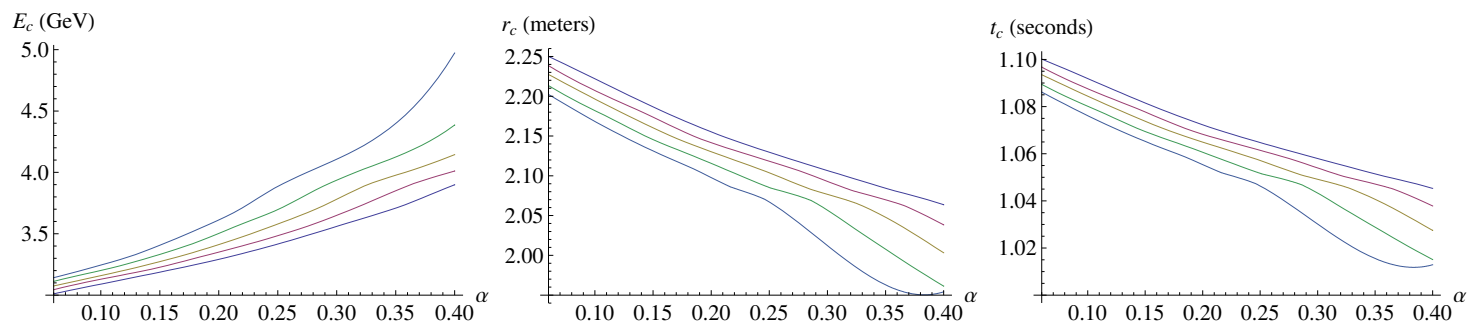

Figure 23. The optimal cylindrical veto parameters as a function of the tracking efficiency parameters $\alpha$ and $\beta$. The curves, from top to bottom, correspond to $\beta=0,0.1,0.2,0.3$ and 0.4 except for the left panel where the order is inverted. 

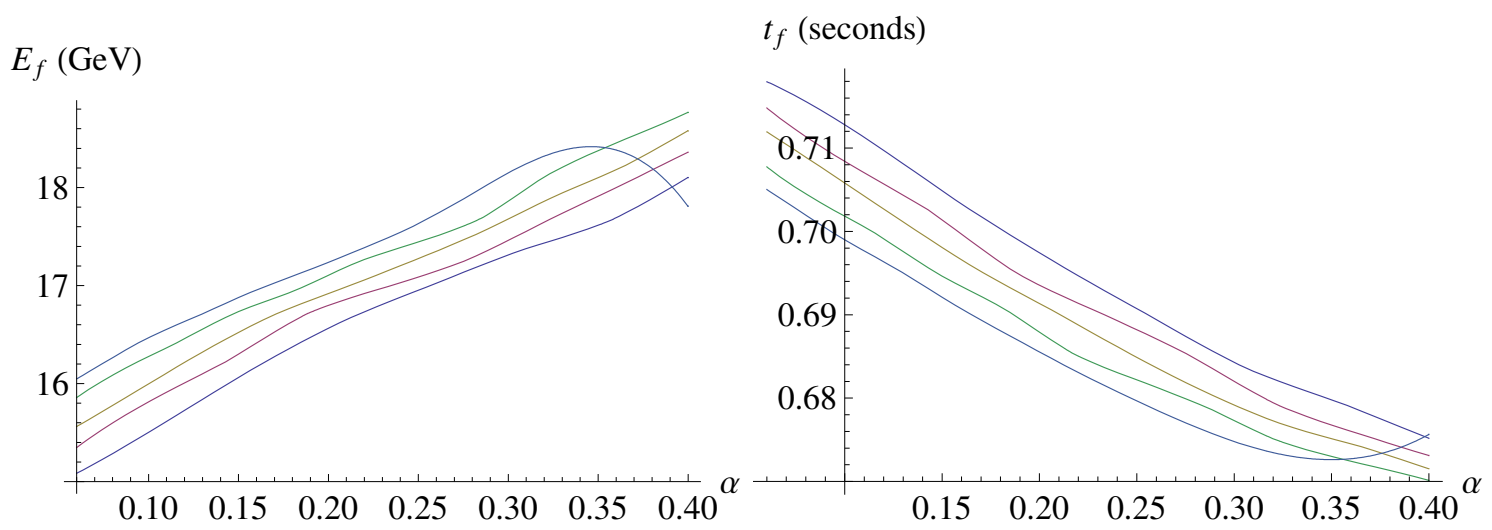

Figure 24. The optimal full detector veto parameters as a function of the tracking efficiency parameters $\alpha$ and $\beta$. The curves in the left (right) panel from bottom to top (top to bottom) correspond to $\beta=0,0.1,0.2,0.3$ and 0.4 .

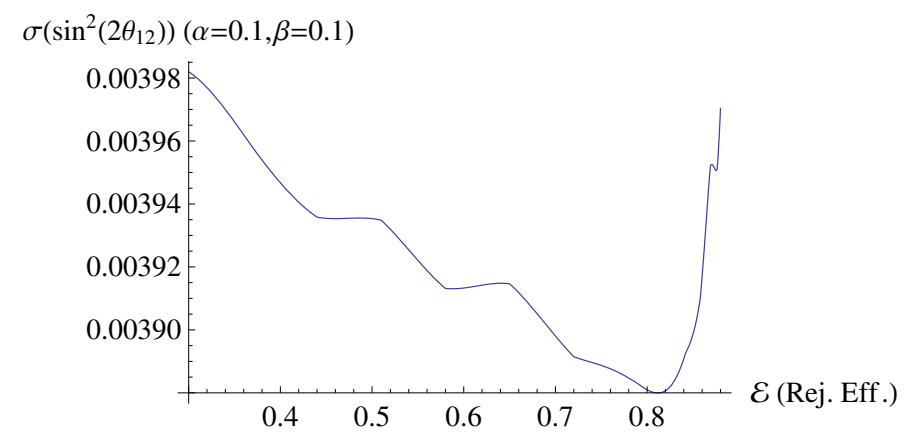

Figure 25. The precision with which $\sin ^{2}\left(2 \theta_{12}\right)$ can be measured as a function of the total rejection efficiency $\mathcal{E}$ with $\alpha=\beta=0.1$.

$\left(\mathcal{E}, t_{l}\right)$ on the curve in figure 17 , corresponding to the maximal live time achievable for each rejection efficiency. This yields figure 25 . Here one can see that with $\alpha=\beta=0.1$, the optimal rejection efficiency is $81 \%$ and it yields a $\sigma\left(\sin ^{2}\left(2 \theta_{12}\right)\right)$ of 0.0039 or $0.46 \%$, as compared with $0.44 \%$ with no background or $0.45 \%$ with perfect tracking. The corresponding optimal veto parameters are summarized in table 3 .

For general tracking efficiency parameters $\alpha$ and $\beta$, one can follow this same strategy to optimize the veto strategy. The corresponding statistic $\sigma\left(\sin ^{2}\left(2 \theta_{12}\right)\right)$ is presented in figure 26. One sees that the precision with which $\theta_{12}$ can be determined is essentially independent of the tracking. By performing the same analysis as in the case of the hierarchy, we have also found that the optimal veto strategy is also quite insensitive to the tracking, and so for brevity we will not repeat the optimal strategy here in general. In the case $\alpha=\beta=0.1$ the optimal strategy is summarized in table 3 .

\section{Conclusions}

The next generation of medium baseline neutrino experiments have two main goals, the determination of the neutrino mass hierarchy and a precise measurement of $\theta_{12}$. The 


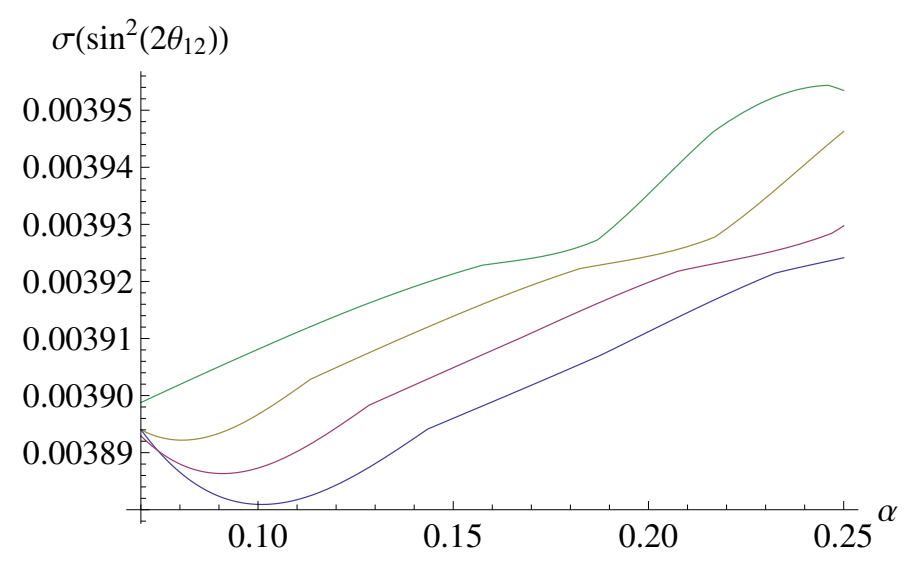

Figure 26. The precision with which $\sin ^{2}\left(2 \theta_{12}\right)$ can be measured as a function of the fraction $\beta$ of muons which are known to be poorly tracked and the fraction $\alpha$ of muons which appear to be well tracked but in fact are poorly tracked. The curves, from bottom to top, correspond to $\beta=0.1,0.2,0.3$ and 0.4 .

\begin{tabular}{|c|l|l|l|l|l|l|l|l|l|l|}
\hline Goal & Stat & $\mathcal{E}$ & $t_{l}$ & $\mathcal{E}_{c}$ & $\mathcal{E}_{f}$ & $E_{c}$ & $t_{c}$ & $r_{c}$ & $E_{f}$ & $t_{f}$ \\
\hline $\mathrm{MH}$ & 11.5 & $78 \%$ & $97 \%$ & $89 \%$ & $66 \%$ & $3.1 \mathrm{GeV}$ & $1.1 \mathrm{~s}$ & $2.2 \mathrm{~m}$ & $16 \mathrm{GeV}$ & $0.7 \mathrm{~s}$ \\
\hline $\sin ^{2}\left(2 \theta_{12}\right)$ & $0.46 \%$ & $81 \%$ & $95 \%$ & $92 \%$ & $73 \%$ & $2.5 \mathrm{GeV}$ & $1.1 \mathrm{~s}$ & $2.3 \mathrm{~m}$ & $12 \mathrm{GeV}$ & $0.8 \mathrm{~s}$ \\
\hline
\end{tabular}

Table 3. The optimal cylindrical veto parameters for the determination of the mass hierarchy and $\theta_{12}$ assuming tracking parameters $\alpha=\beta=0.1$ corresponding to $20 \%$ of muons being poorly tracked of which half are known to be poorly tracked. The statistics reported are as in table 1.

challenges involved in these two goals are different. The first requires a precise measurement of the energies of the 15th, 16th and 17 th $1-3$ oscillation peaks in the reactor neutrino spectrum [31]. These are small and contain few events, therefore statistical fluctuations will be quite important. The second instead requires a measurement of the depth of the solar oscillation maximum, a broad feature which extends across the spectrum visible at these experiments. This feature includes essentially all of the events in the sample, and so it will be measured extremely precisely at these experiments, with an uncertainty which is dominated by a systematic uncertainty in the reactor neutrino spectrum [29].

As statistical fluctuations play a central role in the determination of the hierarchy but not in the measurement of $\theta_{12}$, the optimal ${ }^{9} \mathrm{Li}$ veto strategies are quite different for these two science goals. The first is optimized with a very loose veto, which even with perfect tracking has an efficiency of only $90 \%$. Of course, given the high rate of muon bundles and showering muons [7], the tracking will not be perfect and so the efficiency will be appreciably lower. On the other hand, as statistical fluctuations play a secondary role in the measurement of $\theta_{12}$, a tighter veto is optimal, with an efficiency as high as $93 \%$ in the idealized case of perfect tracking. This is not a result of a large impact of the spallation background on the precision of the measurement of $\theta_{12}$. Indeed, with the $1 \%$ uncertainty assumed here in the spallation background rate, even with no veto at all the precision in the measurement of $\sin ^{2}\left(2 \theta_{12}\right)$ would only increase from $0.44 \%$ to $0.47 \%$. On the contrary, it 
is a result of the fact that a 6 year run is more than is necessary for a precise measurement of $\theta_{12}$, and so additional dead time has little impact on the precision.

Indeed, a smaller detector with a less PMT coverage could measure $\theta_{12}$ nearly as well. More to the point, given the central role of systematic errors, we suspect [16] that the most precise measurement of $\theta_{12}$ at the same price would be given by two smaller detectors, with low PMT coverage, at sufficiently distinct baselines so as to break the degeneracy between the unknown reactor spectrum and $\theta_{12}$. Even if this would preclude a determination of the hierarchy, it may be the option for RENO 50 which would maximize synergy with JUNO and open the door to the measurement of the leptonic CP-violating phase in the years beyond [33].

\section{Acknowledgments}

JE and EC are supported by NSFC grant 11375201. XZ is supported in part by NSFC grants 11121092, 11033005 and 11375202. EC is also supported by the Chinese Academy of Sciences President's International Fellowship Initiative grant 2015PM063. MG is supported by the Chinese Academy of Sciences President's International Fellowship Initiative grant 2015 PM007.

Open Access. This article is distributed under the terms of the Creative Commons Attribution License (CC-BY 4.0), which permits any use, distribution and reproduction in any medium, provided the original author(s) and source are credited.

\section{References}

[1] Y.-F. Li, J. Cao, Y. Wang and L. Zhan, Unambiguous determination of the neutrino mass hierarchy using reactor neutrinos, Phys. Rev. D 88 (2013) 013008 [arXiv:1303.6733] [INSPIRE].

[2] S.-B. Kim, New results from RENO and prospects with RENO-50, arXiv:1412.2199 [INSPIRE].

[3] A. Bandyopadhyay, S. Choubey and S. Goswami, Exploring the sensitivity of current and future experiments to $\theta_{\text {solar }}$, Phys. Rev. D 67 (2003) 113011 [hep-ph/0302243] [INSPIRE].

[4] S.T. Petcov and M. Piai, The LMA MSW solution of the solar neutrino problem, inverted neutrino mass hierarchy and reactor neutrino experiments, Phys. Lett. B 533 (2002) 94 [hep-ph/0112074] [INSPIRE].

[5] S. Choubey, S.T. Petcov and M. Piai, Precision neutrino oscillation physics with an intermediate baseline reactor neutrino experiment, Phys. Rev. D 68 (2003) 113006 [hep-ph/0306017] [INSPIRE].

[6] F. Reines and C.L. Cowan, A proposed experiment to detect the free neutrino, Phys. Rev. 90 (1953) 492 [INSPIRE].

[7] M. Grassi, J. Evslin, E. Ciuffoli and X. Zhang, Showering cosmogenic muons in a large liquid scintillator, JHEP 09 (2014) 049 [arXiv:1401.7796] [INSPIRE]. 
[8] KamLAND collaboration, S. Abe et al., Production of radioactive isotopes through cosmic muon spallation in KamLAND, Phys. Rev. C 81 (2010) 025807 [arXiv:0907.0066] [INSPIRE].

[9] D.A. Dwyer, Precision measurement of neutrino oscillation parameters with KamLAND, Ph.D. thesis, http://kamland.lbl.gov/Dissertations/DanDwyer-DoctorThesis.pdf, UC Berkeley, U.S.A. Spring 2007.

[10] KamLAND collaboration, S. Abe et al., Precision measurement of neutrino oscillation parameters with KamLAND, Phys. Rev. Lett. 100 (2008) 221803 [arXiv:0801.4589] [INSPIRE].

[11] X. Qian, A. Tan, W. Wang, J.J. Ling, R.D. McKeown and C. Zhang, Statistical evaluation of experimental determinations of neutrino mass hierarchy, Phys. Rev. D 86 (2012) 113011 [arXiv:1210.3651] [INSPIRE].

[12] E. Ciuffoli, J. Evslin and X. Zhang, Confidence in a neutrino mass hierarchy determination, JHEP 01 (2014) 095 [arXiv: 1305.5150] [INSPIRE].

[13] J. Evslin, Confidence in the neutrino mass hierarchy, arXiv:1310.4007 [INSPIRE].

[14] E. Ciuffoli, J. Evslin and X. Zhang, Mass hierarchy determination using neutrinos from multiple reactors, JHEP 12 (2012) 004 [arXiv:1209. 2227] [INSPIRE].

[15] X. Qian, D.A. Dwyer, R.D. McKeown, P. Vogel, W. Wang and C. Zhang, Mass hierarchy resolution in reactor anti-neutrino experiments: parameter degeneracies and detector energy response, Phys. Rev. D 87 (2013) 033005 [arXiv:1208.1551] [INSPIRE].

[16] E. Ciuffoli, J. Evslin, Z. Wang, C. Yang, X. Zhang and W. Zhong, Advantages of multiple detectors for the neutrino mass hierarchy determination at reactor experiments, Phys. Rev. D 89 (2014) 073006 [arXiv: 1308.0591] [INSPIRE].

[17] S.B. Kim, Observation of reactor antineutrino disappearance at RENO, talk given at Neutrino 2012, Kyoto Japan June 42012.

[18] RENO collaboration, S.-H. Seo, New results from RENO and the $5 \mathrm{MeV}$ excess, AIP Conf. Proc. 1666 (2015) 080002 [arXiv: 1410.7987] [INSPIRE].

[19] H. de Kerret, Results from Double Chooz, talk given at Neutrino 2014, Boston U.S.A. June 3 2014.

[20] DaYa BAY collaboration, D.V. Naumov, Recent results from Daya Bay experiment, EPJ Web Conf. 95 (2015) 04043 [arXiv: 1412.7806] [INSPIRE].

[21] ISOLDE collaboration, G. Nyman et al., The beta decay of ${ }^{9} \mathrm{Li}$ to levels in ${ }^{9} \mathrm{Be}$ : a new look, Nucl. Phys. A 510 (1990) 189 [InSPIRE].

[22] P.R. Christensen and C.L. Cocke, Neutron decay of the $2.43 \mathrm{MeV}$ and $3.03 \mathrm{MeV}$ states in ${ }^{9} \mathrm{Be}$ to ${ }^{8}$ Be, Nucl. Phys. 89 (1966) 656.

[23] C.L. Cocke and P.R. Christensen, Neutron widths in ${ }^{9}$ Be, Nucl. Phys. A 111 (1968) 623.

[24] T.A.D. Brown et al., Decay studies for states in ${ }^{9} \mathrm{Be}$ up to $11 \mathrm{MeV}$ : insights into the $n+{ }^{8} \mathrm{Be}$ and $\alpha+{ }^{5}$ He cluster structure, Phys. Rev. C 76 (2007) 054605 [INSPIRE].

[25] A. Bandyopadhyay, S. Choubey, S. Goswami and S.T. Petcov, High precision measurements of $\theta_{\text {solar }}$ in solar and reactor neutrino experiments, Phys. Rev. D 72 (2005) 033013 [hep-ph/0410283] [INSPIRE]. 
[26] S.-F. Ge, K. Hagiwara, N. Okamura and Y. Takaesu, Determination of mass hierarchy with medium baseline reactor neutrino experiments, JHEP 05 (2013) 131 [arXiv:1210.8141] [INSPIRE].

[27] A.B. Balantekin et al., Neutrino mass hierarchy determination and other physics potential of medium-baseline reactor neutrino oscillation experiments, arXiv:1307.7419 [INSPIRE].

[28] P. Huber, On the determination of anti-neutrino spectra from nuclear reactors, Phys. Rev. C 84 (2011) 024617 [Erratum ibid. C 85 (2012) 029901] [arXiv: 1106.0687] [INSPIRE].

[29] E. Ciuffoli, J. Evslin, M. Grassi and X. Zhang, Measuring $\theta_{12}$ despite an uncertain reactor neutrino spectrum, arXiv:1503.05126 [INSPIRE].

[30] H. Nunokawa, S.J. Parke and R. Zukanovich Funchal, Another possible way to determine the neutrino mass hierarchy, Phys. Rev. D 72 (2005) 013009 [hep-ph/0503283] [INSPIRE].

[31] E. Ciuffoli, J. Evslin and X. Zhang, The neutrino mass hierarchy at reactor experiments now that $\theta_{13}$ is large, JHEP 03 (2013) 016 [arXiv: 1208.1991] [INSPIRE].

[32] L.A. Winslow, First solar neutrinos from KamLAND: a measurement of the ${ }^{8} B$ solar neutrino flux, Ph.D. thesis, http://kamland.lbl.gov/Dissertations/LindleyWinslow-DoctorThesis.pdf, UC Berkeley, U.S.A. Fall 2008 [ISBN:9781109098198].

[33] E. Ciuffoli, J. Evslin and X. Zhang, The leptonic CP phase from muon decay at rest with two detectors, JHEP 12 (2014) 051 [arXiv:1401.3977] [INSPIRE]. 